\title{
HOPF BIFURCATION OF TRAVELLING PULSES IN SOME BISTABLE REACTION-DIFFUSION SYSTEMS*
}

\author{
TSUTOMU IKEDA ${ }^{\dagger}$, HIDEO IKEDA ${ }^{\ddagger}$, AND MASAYASU MIMURA ${ }^{\S}$
}

\begin{abstract}
The present paper is devoted to the study of the global structure of travelling pulse solutions of a bistable reaction-diffusion system with a sufficiently small parameter $\epsilon>0$. The simplification of piecewise-linear nonlinearity enables us to reveal the global branch of travelling pulse solutions. Using the singular limit analysis as $\epsilon \downarrow 0$, the appearance of the Hopf bifurcation point on the branch is shown, from which propagating pulse solutions with oscillating layers (travelling breathers) arise numerically.
\end{abstract}

1. Introduction. Reaction-diffusion systems have been utilized for describing various nonlinear phenomena and have contributed to theoretical understanding of the mechanism of spatio-temporal pattern formation in diverse fields of natural sciences, for instance, chemical reactions, population dynamics, morphogenesis and combustion. The present paper is devoted to the study of pulse-like patterns among others. Typical experimental examples are excitingly beautiful chemical waves such as target and spiral waves arising in the Belouzov-Zhabotinsky reaction, which have been intensively studied from various points of view ([4], [15], [21] and [22] for instance). Such patterns come into view in two-dimensional media, however, their fundamental mechanism can be explained by the analysis of pulse solutions on the infinite line. It is already shown in some mathematical models that there are stable pulse solutions propagating on the infinite line with non-zero constant velocity (travelling pulses, [3], [8] and [14]) and stable motionless pulse solutions (standing pulses, [2], [3], [5] and $[10])$. Moreover, as numerical evidences, there are stable layer-oscillating pulse solutions (in-phase breathers, [13] and [19]) as the consequence of the Hopf bifurcation from standing pulses. Recently, as shown in Figures 1.1 and 1.2, stable layer-oscillating propagating pulse solutions called travelling breathers have been found in numerical simulations ([12]) for the following reaction-diffusion system:

$$
u_{t}=u_{x x}+f(u, v), \quad v_{t}=D v_{x x}+g(u, v) \quad \text { for } x \in(0, L) \text { and } t>0
$$

subject to the periodic boundary condition on both the activator $u(t, x)$ and inhibitor $v(t, x)$. Here, $L \gg 1$ is fixed, $D$ is a positive parameter and the nonlinearity $\{f, g\}$ is given by

$$
f(u, v)=u(u-\alpha)(\beta-u)-v \text { and } g(u, v)=\delta(u-\gamma v)
$$

with positive constants $\alpha, \beta(\alpha<\beta), \gamma$ and $\delta \ll 1$. When $\{f, g\}$ is far from the odd symmetry, no travelling breather can be observed for any $D>0$ although travelling pulses, standing pulses and in-phase breathers come into view for suitable values of $D$ (A nonlinearity $\{f, g\}$ is said to be odd symmetric if there exists a point $\left(u_{0}, v_{0}\right)$ such that $f\left(u_{0}, v_{0}\right)=g\left(u_{0}, v_{0}\right)=0, f\left(u_{0}+u, v_{0}+v\right)=-f\left(u_{0}-u, v_{0}-v\right)$ and

\footnotetext{
*Received Feb 24, 1999; revised Oct. 18, 1999.

† Department of Applied Mathematics and Informatics, Ryukoku University, Ohtsu 520-2194, JAPAN (tsutomu@rins. ryukoku.ac.jp).

$\ddagger$ Department of Mathematics, Toyama University, Toyama 930-8555, JAPAN.

$\S$ Institute for Nonlinear Sciences and Applied Mathematics, Hiroshima University, HigashiHiroshima 739-8526, JAPAN.
} 


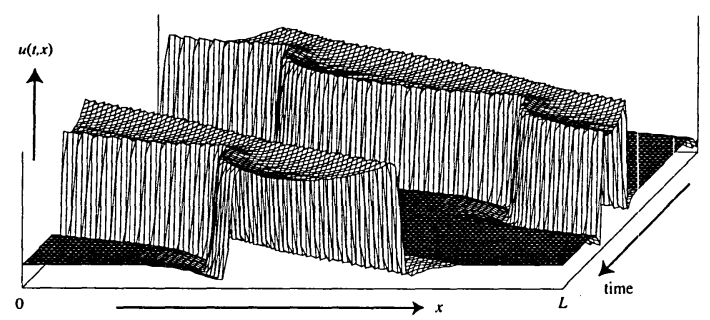

FIG. 1.1. Profiles of travelling breathers of (1.1)

$g\left(u_{0}+u, v_{0}+v\right)=-g\left(u_{0}-u, v_{0}-v\right)$ for any $\left.(u, v) \in \mathbf{R}^{2}\right)$. When $\{f, g\}$ becomes close to the odd symmetry, on the other-hand, the following different behavior is observed. The system (1.1) has a stable travelling pulse for small $D$. As $D$ increases, the travelling pulse loses its stability and gives place to a travelling breather. Figures 1.1 and 1.2 exhibit respectively profiles and contour lines of $u(t, x)$ calculated by the conventional implicit finite difference method for (1.1) with $L=400.0, \alpha=0.112635$, $\beta=1.005256, \gamma=6.0, \delta=0.002$ and $D=3.51$ (the spatial mesh size $\Delta x=0.5$ and the time increment $\Delta t=0.1$ ).

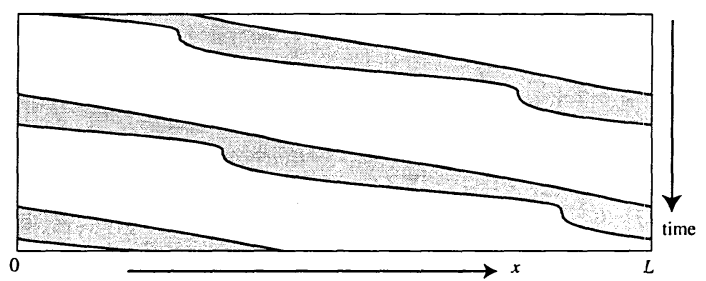

FIG. 1.2. Travelling breathers of (1.1) (excited regions are shaded)

By analogy with the advent of in-phase breathers from standing pulses through the Hopf bifurcation, we reason that the Hopf bifurcation of travelling pulses brings about travelling breathers. Since travelling breathers may exist only when the nonlinearity is close to the odd symmetry, the present paper deals with bistable systems in the following form:

$$
\epsilon \tau u_{t}=\epsilon^{2} u_{x x}+f(u, v), \quad v_{t}=v_{x x}+g(u, v) \quad \text { for } x \in \mathbf{R} \text { and } t>0,
$$

where both parameters $\epsilon$ and $\tau$ are positive and $\epsilon$ is assumed to be small. Since $\epsilon$ expresses the width of the transition layer and $\tau$ controls the relaxation-time ratio, they are called layer and relaxation parameters, respectively. We note that putting $\epsilon=\sqrt{\delta / D}, \tau=\sqrt{\delta D}$ and replacing $x$ and $t$ respectively by $\epsilon x$ and $\delta t$, we can rewrite (1.1) as (1.2). The system (1.2) is assumed to have two stable spatially uniform equilibria $\left(u_{-}, v_{-}\right)$and $\left(u_{+}, v_{+}\right)$as shown in Figure $1.3\left(u_{-}<u_{0}<u_{+}\right)$. Precise assumptions on the nonlinearity $\{f, g\}$ are stated in (1) of Appendix, and we here note only that the nullcline of $f$ consists of three continuous curves $u=h_{-}(v), u=h_{0}(v)$ and $u=h_{+}(v)$. For smooth initial data, the diffusion term $\epsilon^{2} u_{x x}$ is negligible for some time interval, so that (1.2) is approximated by

$$
\epsilon \tau u_{t}=f(u, v), \quad v_{t}=v_{x x}+g(u, v) .
$$

Since $f$ takes cubic nonlinearity and $\epsilon$ is small, the line $\mathbf{R}$ is decomposed into two regions, say, $u \cong h_{-}(v)$ (rest state) in one region while $u \cong h_{+}(v)$ (excited state) in 
the other. Thus, there appear several transition layers of the width of $O(\epsilon)$ in the profile of $u(t, x)$. Since we are interested in travelling breathers shown in Figures 1.1 and 1.2 , we focus our attention on pulse solutions of (1.2) having exactly one localized excited region.

We hope to clarify the appearance of stable travelling breathers from travelling pulses through the Hopf bifurcation, and in the present paper

- we reveal the global branch of travelling and standing pulses of (1.2) with small $\epsilon>0$ and the piecewise-linear nonlinearity (1.3),

- we show the existence of the Hopf bifurcation point on the branch of travelling pulses of the singular limit system (1.4) derived from (1.2) in the limit of $\epsilon \downarrow 0$, and

- we confirm numerically the appearance of stable travelling breathers of (1.2) with small $\epsilon>0$ from a critical point corresponding to the above Hopf bifurcation point.

Generally speaking, the system (1.2) prefers steady states and has stable motionless solutions if $\tau$ is very large (i.e. $\tau=O(1 / \epsilon)$ ) while (1.2) has stable propagating wave solutions if $\tau$ is very small (i.e. $\tau=O(\epsilon)$ ) as was pointed out by Nishiura [18]. Actually, it is proved that (1.2) has a standing pulse which is stable for large $\tau$. Furthermore, it has been shown ([8], [9] and [14]) that when $\{f, g\}$ is slightly perturbed from the odd symmetry, (1.2) has a pair of stable travelling pulses with velocity $\pm s$ and an unstable standing pulse for small $\tau$. This information suggests that the travelling pulses bifurcate from the standing pulse as shown in Figure 1.4. When we assign each pulse its stability symbol $\left\{\sigma_{0}, \sigma_{1}, \sigma_{2}, \sigma_{3}\right\}, \sigma_{i}=0,-$ or + , that means the signs of real parts of four critical eigenvalues of the linearized problem around the pulse, we know that the standing pulse has no unstable eigenvalues for large $\tau$ while it has three unstable ones for small $\tau$. This change indicates that high degeneracy occurs around the singular point on the branch of standing pulses.

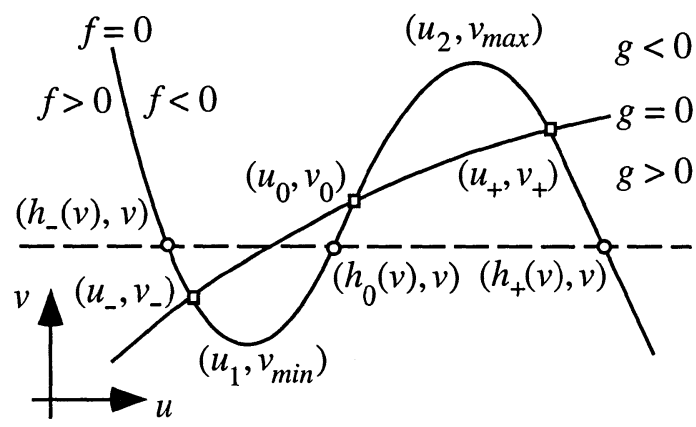

FIG. 1.3. Bistable nonlinearity

In the companion volume [10], it is found that the stable standing pulse is destabilized through both the Hopf and static bifurcations as $\tau$ decreases. Thus, the stability dependence of standing pulse on $\tau$ is clarified. That is, the stable standing pulse loses its stability via both the Hopf bifurcation at $P_{H}^{\epsilon}$ and the static bifurcations at $P_{\text {static }}^{\epsilon}$ as $\tau$ decreases and therefore it has three unstable eigenvalues for small $\tau$ (Figure 1.5).

The bifurcation picture of pulse solutions could be simply explained if the static bifurcation from the standing pulse could occur primarily and super-critically before the Hopf bifurcation occurs as $\tau$ decreases. That is, a travelling pulse resulting from the static bifurcation could keep its stability for any small $\tau$. However, this assumption is not true. The order that the Hopf and static bifurcation points appear depends on the nonlinearity $\{f, g\}$ ([10], [12] and [13]). 


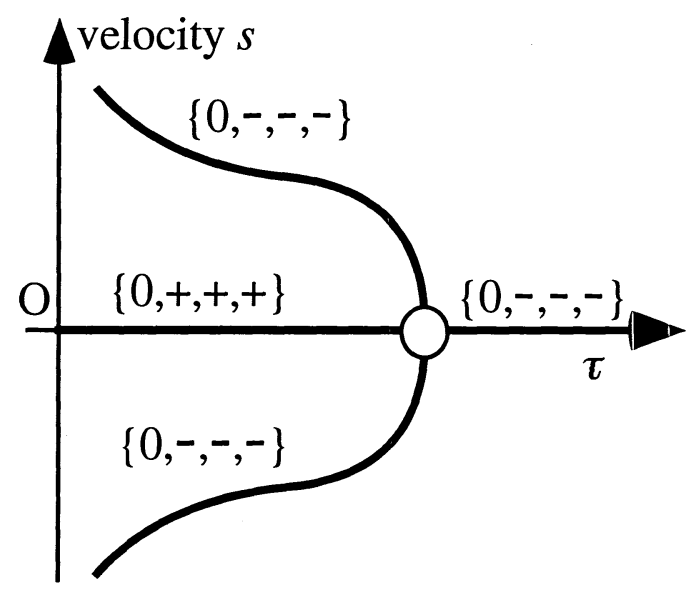

FIG. 1.4. Velocity s of travelling pulses

We here consider the forthcoming piecewise-linear nonlinearity (1.3) with $0<$ $d \ll 1$, which is close to the odd symmetry. Then, the stable standing pulse has lost its stability at $P_{H}^{\epsilon}$ first and no stable solution can arise from $P_{\text {static }}^{\epsilon}$. It is also proved that the travelling pulse is stable for small $\tau$. Hence, if the branches of travelling pulses and standing pulses are connected at $P_{\text {static }}^{\epsilon}$, the bifurcation theory suggests that the branch of travelling pulses contains the Hopf bifurcation point $P_{H o p f}^{\epsilon}$, and moreover, a travelling breather may bifurcate stably at $P_{\text {Hopf }}^{\epsilon}$ if the stable travelling pulse is first destabilized at $P_{H o p f}^{\epsilon}$ as $\tau$ increases. In the present paper, combining analytical and numerical methods, we show that a travelling breather appears along the above story.

It may be possible to give a formal scheme like (2.8) in Section 2 that determines the branch of travelling pulses for a class of general nonlinearities. However, the construction of the branch is not easy and the stability analysis along the branch is much more difficult. Actually, the existence of stable travelling pulses is assured only when the nonlinearity is very close to the odd symmetry ([8] and [9]), and the order of the Hopf and static bifurcation points is known for some special nonlinearities alone ([10]).

With a view to discussing the existence and stability of travelling pulses in detail and getting a global bifurcation picture of pulse solutions, we adopt the following McKean type nonlinearity [16] as a prototype in the present paper:

$$
f(u, v)=\left\{\begin{array}{ll}
-u-1-v / p & \text { if } u<0 \\
-u+1-v / p & \text { if } u>0
\end{array}, \quad g(u, v ; d)=u+d-(1-1 / p) v\right.
$$

where $p \geq 2$ is a constant. The relative attraction of two stable equilibria

$$
\left(u_{-}, v_{-}\right)=\left(-\frac{p+d-1}{p}, d-1\right) \text { and }\left(u_{+}, v_{+}\right)=\left(\frac{p-d-1}{p}, d+1\right)
$$

is controlled by $d \in(1-p, p-1)$. If $d=0$, the nonlinearity becomes odd symmetric and two equilibria have the same attraction. If $d>0$, then $\left(u_{-}, v_{-}\right)$is more attractive and (1.2) has standing and travelling pulse solutions with localized excited region. We 
suitably fix $p \geq 2$ and adopt $d \in[0, p-1)$ as a parameter since we deal with the case where the excited region is localized.

Here, let us indicate intuitively how we obtain the singular limit system (1.4) below from the reaction-diffusion system (1.2). We deal with pulse solutions of (1.2) having two transition layers such that the excited state is localized between the layers. Since $u \cong h_{-}(v)$ in the rest region and $u \cong h_{+}(v)$ in the excited region, the second equation of (1.2) is approximated by

$v_{t}=v_{x x}+G_{-}(v)$ in the rest region, $\quad v_{t}=v_{x x}+G_{+}(v)$ in the excited region

when $\epsilon$ is small, where $G_{-}(v) \equiv g\left(h_{-}(v), v\right)$ and $G_{+}(v) \equiv g\left(h_{+}(v), v\right)$. Let $x=\psi_{+}^{\epsilon}\left(t_{*}\right)$ be the center of the right internal layer at time $t_{*}$ and put $v_{*}=v\left(t_{*}, \psi_{+}^{\epsilon}\left(t_{*}\right)\right)$. When $\epsilon$ is small, the first equation of (1.2) is approximated by $\epsilon \tau u_{t}=\epsilon^{2} u_{x x}+f\left(u, v_{*}\right)$ in a neighborhood of $\left(t_{*}, \psi_{+}^{\epsilon}\left(t_{*}\right)\right)$, which is rewritten as

$$
\tilde{u}_{s}=\tilde{u}_{y y}+f\left(\tilde{u}, v_{*}\right)
$$

by the introduction of stretched variables $y=\left(x-\psi_{+}^{\epsilon}\left(t_{*}\right)\right) / \epsilon$ and $s=t / \epsilon \tau$, where $\tilde{u}(s, y)=u\left(\epsilon \tau s, \epsilon y+\psi_{+}^{\epsilon}\left(t_{*}\right)\right)$. If $v_{*} \in\left(v_{\min }, v_{\max }\right)$, the above scalar reaction-diffusion equation subject to

$$
\lim _{y \downarrow-\infty} \tilde{u}(s, y)=h_{+}\left(v_{*}\right) \text { and } \lim _{y \uparrow \infty} \tilde{u}(s, y)=h_{-}\left(v_{*}\right)
$$

has a travelling wave solution with velocity $c\left(v_{*}\right)$ depending on $v_{*}$. Thus, when $\epsilon$ is small, the velocity of right internal layer at time $t_{*}$ is approximated by $c\left(v\left(t_{*}, \psi_{+}^{\epsilon}\left(t_{*}\right)\right) / \tau\right.$ and the velocity of left internal layer is also approximated by $-c\left(v\left(t_{*}, \psi_{-}^{\epsilon}\left(t_{*}\right)\right) / \tau\right.$, where $\psi_{-}^{\epsilon}\left(t_{*}\right)$ denotes the center of left layer. We will first study pulses in the limit of $\epsilon \downarrow 0$ where transition layers can be regarded as interfaces, and then extend results to the system (1.2) with small $\epsilon>0$. Let $x=\psi_{-}(t)$ and $x=\psi_{+}(t)$ be two interfaces and assume that the excited state is localized in $\left(\psi_{-}(t), \psi_{+}(t)\right)$. The singular perturbation method gives the following description of the dynamics of interfaces called the singular limit system:

$$
\begin{aligned}
& \left.\begin{array}{rl}
\tau \frac{d \psi_{-}}{d t} & =-c\left(v\left(t, \psi_{-}(t)\right)\right) \\
\tau \frac{d \psi_{+}}{d t} & =c\left(v\left(t, \psi_{+}(t)\right)\right)
\end{array}\right\} \text { for } t>0, \quad v(t, \cdot) \in \mathbf{C}^{\mathbf{1}}(\mathbf{R}) \\
& v_{t}=v_{x x}+G_{-}(v) \text { for } x \notin\left[\psi_{-}(t), \psi_{+}(t)\right] \text {, } \\
& v_{t}=v_{x x}+G_{+}(v) \text { for } x \in\left(\psi_{-}(t), \psi_{+}(t)\right) \text {. }
\end{aligned}
$$

The problem (1.2) (resp. (1.4)) with (1.3) is denoted by (RD) (resp. (SL)) hereafter.

The outline of the paper is as follows. In Section 2, we derive a necessary and sufficient condition for the existence of a travelling pulse of the singular limit system (1.4) with general nonlinearities satisfying (A1) (A4) stated in Appendix. In Sections $3 \sim 5$, we fully study pulse solutions by adopting the piecewise linear nonlinearity (1.3). Section 3 reviews briefly the existence and stability of travelling front/back waves and standing pulses of (RD) and (SL) after discussing the range of parameters $p$ and $d$ included in (1.3). Section 4 constructs travelling pulses of (SL). We show the unique existence of the branch of travelling pulses, the connection by the branch between the bifurcation point from travelling front/back waves and the static bifurcation point from standing pulses, the direction of static bifurcation and so on. Section 5 investigates the behavior of four critical eigenvalues of the formal linearized problem of (SL) 
around travelling pulses along the branch. All propositions in Sections 4 and 5 are proved in Appendix. Even for (1.3), however, rigorous treatments are not sufficient for deriving more detailed information such as the numbers of turning points and the Hopf bifurcation points on the branch of travelling pulses. Since (1.3) is piecewise linear, the branch is determined by a transcendental equation and the critical eigenvalues are solutions of another transcendental equation. One of reasonable approaches is to numerically solve these equations with high accuracy. Section 6 shows the global bifurcation picture of pulse solutions of (SL) obtained by combining the theoretical and numerical results. Several remarks and future problems are mentioned in Section 7.

Our assertion consists of two parts: one is about the global branch of travelling pulses of (RD) and the other is about the appearance of the Hopf bifurcation from the branch of travelling pulses of (SL). The following theorems on the existence of branches of travelling pulses are obtained by extending Propositions 1, 2, 4, 5 and 8 for the singular limit system (SL) to the reaction-diffusion system (RD) with small $\epsilon$. The proof of the theorem is omitted since it is similar to those of Theorems 2.7, 3.10, 3.17 and 3.18 in [10] clarifying the existence and stability of standing pulses of (RD) with small $\epsilon$ based on the theory in Henry [6].

Theorem 1.1. Fix $d \in(0,1)$. There exists $\epsilon_{0}>0$ such that the following holds for $\epsilon<\epsilon_{0}$.

(1) Let $\tau_{H}^{\epsilon}(d)$ and $\tau_{\text {static }}^{\epsilon}(d)$ be respectively the Hopf and static bifurcation points of the standing pulse of $(R D)$. Then $\tau_{H}^{\epsilon}(d)>\tau_{\text {static }}^{\epsilon}(d)$ if $d$ is near 0 , while $\tau_{\text {static }}^{\epsilon}(d)>\tau_{H}^{\epsilon}(d)$ if $d$ is near 1.

(2) There exists a unique smooth function $\tau_{d}^{\epsilon}(s)$ on $(0, \infty)$ such that $(R D)$ has a travelling pulse with the velocity $s$ for almost all $s>0$ if $\tau=\tau_{d}^{\epsilon}(s)$. The travelling pulse is unique up to phase shifts and it is stable when $s$ is large. Moreover, $\tau_{d}^{\epsilon}(s)$ tends to $\tau_{\text {static }}^{\epsilon}(d)$ as $s \downarrow 0$ while $\lim _{s \uparrow \infty} \tau_{d}^{\epsilon}(s)=0$.

(3) The second derivative of $\tau_{d}^{\epsilon}(s)$ is negative around $s=0$ if $d<d_{c}^{\epsilon}$ while it is positive there if $d>d_{c}^{\epsilon}$, where $d_{c}^{\epsilon}$ is a constant depending on $\epsilon$.

The first statement (1) of the above theorem is already shown in [10] while (2) and (3) are new results and very important to draw the global structure of travelling pulses: (2) is about the global branch of travelling pulses which is bifurcated from $\left(\tau_{\text {static }}^{\epsilon}(0), 0\right)$ and $(3)$ is about the direction of the bifurcation.

We have studied the branch of travelling pulses also by fixing $\tau$ and adopting $d$ as a bifurcation parameter. The existence of stable travelling pulses has been shown only for $d \simeq 0$ in [8] and [9], so that the global bifurcation diagram of pulse solutions in the $(d, s)$-plane is first given in Theorem 1.2 below. The existence and stability of standing pulses are given in the first statement (1), where the destabilization of standing pulses is described in a complicated form since the inverse function of $\tau_{H}^{\epsilon}(d)$ is multi-valued as revealed in Figure 3.1. The second statement (2) clarifies the connection by the branch of travelling pulses between the static bifurcation point $\left(d_{\text {static }}^{\epsilon}(\tau), 0\right)$ from standing pulses on the $d$-axis and the bifurcation point $\left(s_{*}^{\epsilon}(\tau), 0\right)$ from travelling front/back waves on the $s$-axis. The third one (3) is about the direction of the static bifurcation. (For the bifurcation point from travelling front/back waves, see Fig. 11 of [8], Fig. 4 of [9] or Fig. 4.2 of [14].)

Theorem 1.2. Fix $\tau<1 / p$. There exists $\epsilon_{0}>0$ such that the following holds for $\epsilon<\epsilon_{0}$. 


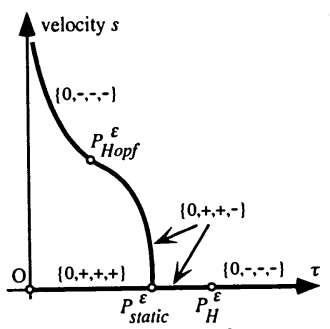

(1) $0<d<d_{c}^{\varepsilon}$

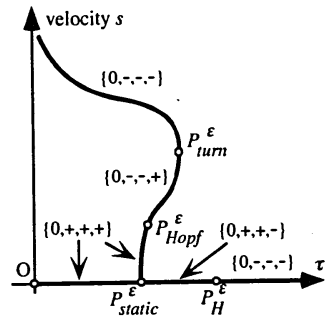

(3) $d_{\text {Hopf }}^{\varepsilon}<d<d_{\text {double }}^{\varepsilon}$

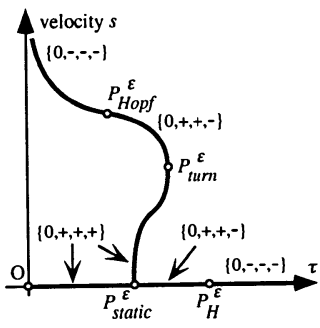

(2) $d_{c}^{\varepsilon}<d<d_{\text {Hopf }}^{\varepsilon}$

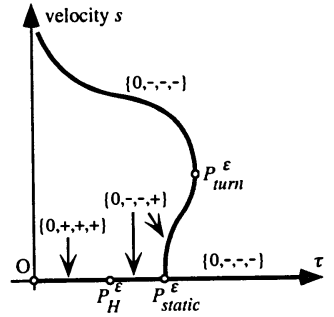

(4) $d_{\text {double }}^{\varepsilon}<d<1$

FIG. 1.5. Global bifurcation pictures of standing and travelling pulses of (RD) (d: fixed)

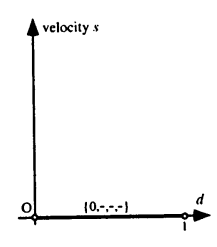

(1) $r>\tau_{\text {peak }}^{\varepsilon}$

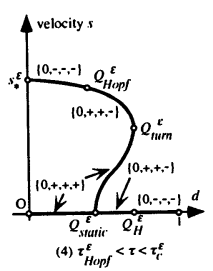

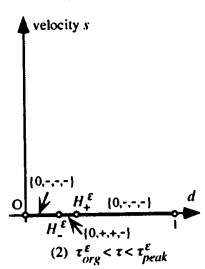

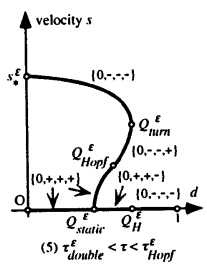

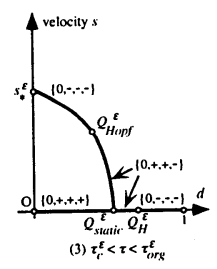

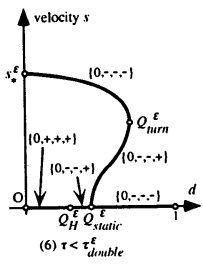

FIG. 1.6. Global bifurcation pictures of standing and travelling pulses of (RD) ( $\tau$ : fixed)

(1) (RD) has a standing pulse for each $d \in(0,1)$, which is unique up to phase shifts. It is stable for large $d$, and is destabilized through the Hopf and static bifurcations as $d$ decreases. Let $d_{H}^{\epsilon}(\tau)$ be the first Hopf bifurcation point and $d_{\text {static }}^{\epsilon}(\tau)$ be the static bifurcation point. Then $d_{H}^{\epsilon}(\tau)>d_{\text {static }}^{\epsilon}(\tau)$ if $\tau$ is near $1 / p$ while $d_{\text {static }}^{\epsilon}(\tau)>d_{H}^{\epsilon}(\tau)$ if $\tau$ is near 0 .

(2) There exists a unique smooth function $d_{\tau}^{\epsilon}(s)$ on $\left(0, s_{*}^{\epsilon}(\tau)\right)$ such that $(R D)$ has a travelling pulse with the velocity $s$ for almost all $s \in\left(0, s_{*}^{\epsilon}(\tau)\right)$ if $d=d_{\tau}^{\epsilon}(s)$, where

$$
s_{*}^{\epsilon}(\tau)=(2 / \tau)\left(1-p^{2} \tau^{2}\right)^{1 / 2}\left(p^{2}-1\right)^{-1 / 2}+o(1) \text { as } \epsilon \downarrow 0 .
$$

The travelling pulse is unique up to phase shifts and it is stable when $s$ is near $s_{*}^{\epsilon}(\tau)$. Moreover, $d_{\tau}^{\epsilon}(s)$ tends to $d_{\text {static }}^{\epsilon}(\tau)$ as $s \downarrow 0$ while $\left(d_{\tau}^{\epsilon}(s), s\right)$ tends to the bifurcation point $\left(0, s_{*}^{\epsilon}(\tau)\right)$ from travelling front/back waves as $s \uparrow s_{*}^{\epsilon}(\tau)$. 
(3) The second derivative of $d_{\tau}^{\epsilon}(s)$ is negative around $s=0$ if $\tau>\tau_{c}^{\epsilon} \equiv \tau_{\text {static }}^{\epsilon}\left(d_{c}^{\epsilon}\right)$ while it is positive there if $\tau<\tau_{c}^{\epsilon}$.

The assertions in the above two theorems are derived from the results for (SL) by applying the singular perturbation technique with respect to $\epsilon>0$. Hence, it holds that

$$
\begin{aligned}
& \lim _{\epsilon \downarrow 0} \tau_{H}^{\epsilon}(d)=\tau_{H}(d), \quad \lim _{\epsilon \downarrow 0} \tau_{\text {static }}^{\epsilon}(d)=\tau_{\text {static }}(d), \\
& \lim _{\epsilon \downarrow 0} d_{H}^{\epsilon}(\tau)=d_{H}(\tau), \lim _{\epsilon \downarrow 0} d_{\text {static }}^{\epsilon}(\tau)=d_{\text {static }}(\tau), \\
& \lim _{\epsilon \downarrow 0} \tau_{d}^{\epsilon}(s)=\tau_{d}\left(s\left(s^{2}+4\right)^{-1 / 2}\right), \quad \lim _{\epsilon \downarrow 0} d_{\tau}^{\epsilon}(s)=d_{\tau}\left(s\left(s^{2}+4\right)^{-1 / 2}\right), \\
& \lim _{\epsilon \downarrow 0} s_{*}^{\epsilon}(\tau)=2 S_{*}(\tau)\left(1-S_{*}(\tau)^{2}\right)^{-1 / 2}, \quad \lim _{\epsilon \downarrow 0} d_{c}^{\epsilon}=d_{c} \text { and } \lim _{\epsilon \downarrow 0} \tau_{c}^{\epsilon}=\tau_{c},
\end{aligned}
$$

where $\tau_{H}(d), \tau_{\text {static }}(d), \tau_{d}(S), d_{\tau}(S), S_{*}(\tau), d_{c}$ and $\tau_{c}$ are given in Propositions $2 \sim$ 5 , and $d_{H}(\tau)$ (resp. $d_{\text {static }}(\tau)$ ) is the inverse function of $\tau_{H}(d)$ (resp. $\tau_{\text {static }}(d)$ ) as explained in Section 6. The branch of travelling pulses of (SL) contains some turning points, and we need the phrase 'for almost all $s$ ' in the second assertion of Theorems 1.1 and 1.2 since we cannot extend the results for (SL) to the reaction-diffusion system (RD) with $\epsilon>0$ at such turning points.

The static bifurcation point $P_{\text {static }}(d)=\left(\tau_{\text {static }}(d), 0\right)$ of standing pulses of (SL) is given by the explicit formula (3.6). For (SL), solving the corresponding transcendental equations by the bisection method, we can calculate with very high accuracy the Hopf bifurcation point $P_{H}(d)=\left(\tau_{H}(d), 0\right)$ of the standing pulse, the branch $\left\{\left(\tau_{d}(s), s\right)\right\}$ of travelling pulses, critical real eigenvalues of the formal linearized problem around travelling pulses along the branch and turning points on the branch. Solving a system of transcendental equations by Newton's method, we can also calculate critical complex eigenvalues and the Hopf bifurcation point on the branch with high accuracy. For instance, Figure 3.1 shows the graphs of $\tau_{H}(d)$ and $\tau_{\text {static }}(d)$ with $p=2$, and Figure 6.2 displays the positions of the Hopf bifurcation point and turning point on the branch of travelling pulses. Combining these results obtained by mathematical analysis (Theorems 1.1 and 1.2) and numerical calculations (Conjectures $1 \sim 5$ ), we arrive at the conjecture as shown in Figure 1.5. We here explain the bifurcation pictures (1) $\sim(4)$ in Figure 1.5.

Fix a sufficiently small $\epsilon>0$. For each fixed $d \in(0,1)$ (RD) has a unique branch $\left\{\left(\tau_{d}^{\epsilon}(s), s\right) ; s>0\right\}$ of travelling pulses in the $(\tau, s)$-plane, and there exist constants $d_{\text {Hopf }}^{\epsilon}$ and $d_{\text {double }}^{\epsilon}$ such that the bifurcation picture of standing and travelling pulses becomes as follows.

(1) Fix $d \in\left(0, d_{c}^{\epsilon}\right)$. Then $\tau_{H}^{\epsilon}(d)>\tau_{\text {static }}^{\epsilon}(d)$. A travelling pulse bifurcates supercritically from $P_{\text {static }}^{\epsilon}(d)=\left(\tau_{\text {static }}^{\epsilon}(d), 0\right)$, and it recovers the stability at the Hopf bifurcation point $P_{H o p f}^{\epsilon}(d)$ on the branch of travelling pulses. The branch has no turning point, and $\lim _{d \downarrow 0, \epsilon \downarrow 0} P_{H o p f}^{\epsilon}(d)=(1 / p, 0)$.

(2) Fix $d \in\left(d_{c}^{\epsilon}, d_{H o p f}^{\epsilon}\right)$. Then $\tau_{H}^{\epsilon}(d)>\tau_{\text {static }}^{\epsilon}(d)$ also. A travelling pulse bifurcates sub-critically from $P_{\text {static }}^{\epsilon}(d)$, and it recovers the stability first at a turning point $P_{\text {turn }}^{\epsilon}(d)$ and then at $P_{\text {Hopf }}^{\epsilon}(d)$. The point $P_{\text {turn }}^{\epsilon}(d)$ tends to $P_{\text {static }}^{\epsilon}\left(d_{c}^{\epsilon}\right)$ as $d \downarrow d_{c}^{\epsilon}$.

(3) Fix $d \in\left(d_{\text {Hopf }}^{\epsilon}, d_{d o u b l e}^{\epsilon}\right)$. Then the bifurcation picture is similar to the case (2) except the order between $P_{\text {turn }}^{\epsilon}(d)$ and $P_{\text {Hopf }}^{\epsilon}(d)$. The point $P_{\text {Hopf }}^{\epsilon}(d)$ tends to $P_{\text {static }}^{\epsilon}\left(d_{\text {double }}^{\epsilon}\right)=P_{H}^{\epsilon}\left(d_{\text {double }}^{\epsilon}\right)$ as $d \uparrow d_{\text {double }}^{\epsilon}$. 
(4) Fix $d \in\left(d_{\text {double }}^{\epsilon}, 1\right)$. Then $\tau_{\text {static }}^{\epsilon}(d)>\tau_{H}^{\epsilon}(d)$. A travelling pulse bifurcates subcritically from $P_{\text {static }}^{\epsilon}(d)$, and it recovers the stability at $P_{t u r n}^{\epsilon}(d)$. The branch has no Hopf bifurcation point, and $\lim _{d \uparrow 1} P_{\text {turn }}^{\epsilon}(d)=(0, \infty)$.

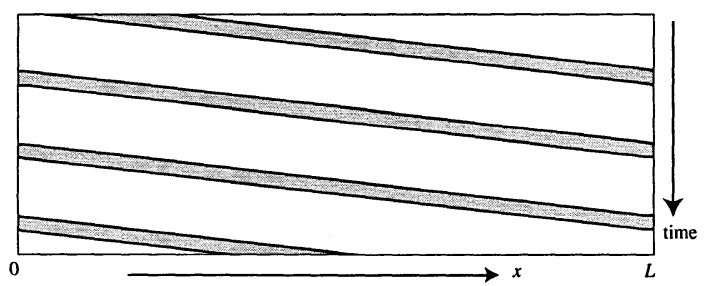

FIG. 1.7. Travelling pulses (excited regions are shaded, $d=0.0160$ )

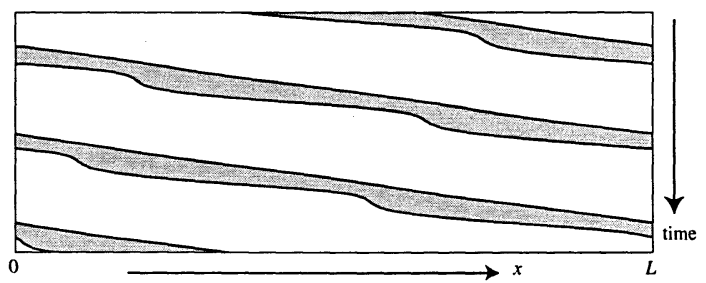

FIG. 1.8. Travelling breathers (excited regions are shaded, $d=0.0170$ )

The transition process of bifurcation pictures becomes clear if we draw pictures in the $(d, s)$-plane by fixing $\tau>0$ and adopting $d$ as a bifurcation parameter. The Hopf and static bifurcation points of standing pulses of (RD) are denoted respectively by $Q_{H}^{\epsilon}(\tau)=\left(d_{H}^{\epsilon}(\tau), 0\right)$ and $Q_{\text {static }}^{\epsilon}(\tau)=\left(d_{\text {static }}^{\epsilon}(\tau), 0\right)$ in Figure 1.6. The transition process is explained in Section 7.

The appearance of stable travelling breathers from travelling pulses via the Hopf bifurcation is numerically shown for the problem (RD) on a finite interval subject to the periodic boundary condition. Figures 1.7 and 1.8 represent the contour lines of numerical solutions $u(t, x)$ of the problem (RD) with $p=2.0, \tau=0.46$ and the length $L=25.0$ of the finite interval. In numerical computations, the spatial mesh size $\Delta x=0.05$ and the time increment $\Delta t=0.005$ are fixed, and $d$ is adopted as a bifurcation parameter. The travelling pulse arising from the bifurcation point of travelling front/back waves (Fig. 11 of [8], Fig. 4 of [9] or Fig. 4.2 of [14]) keeps its stability for small $d$ as shown in Figure 1.7. At a critical value between $d=0.0160$ and 0.0167 the travelling pulse loses its stability, and a stable travelling breather comes into view when $d$ is slightly greater than the critical value as shown in Figure 1.8.

2. A scheme for finding travelling pulses. Let $\{v(z), m ; s\}$ be a travelling pulse of (1.4) with the width $2 m>0$ of excited region and the velocity $s$, where $z=x-s t$ is the moving coordinate. Then, through a suitable phase shift $\{v(z), m ; s\}$ satisfies the following equations:

$$
\begin{aligned}
& \tau s=-c(v(-m)), \quad \tau s=c(v(m)), \quad v \in \mathbf{C}^{\mathbf{1}}(\mathbf{R}), \\
& v^{\prime \prime}+s v^{\prime}+G_{-}(v)=0 \text { for } z \notin[-m, m], \\
& v^{\prime \prime}+s v^{\prime}+G_{+}(v)=0 \text { for } z \in(-m, m)
\end{aligned}
$$


subject to the boundary condition $\lim _{|z| \uparrow \infty} v(z)=v_{-}$. We may assume $s \geq 0$ since if $\{v(z), m ; s\}$ is a travelling pulse of $(1.4)$ so is $\{v(-z), m ;-s\}$. Put $w=\bar{v}^{\prime}$. Then, $(v(z), w(z))$ satisfies

$$
\begin{gathered}
\lim _{|z| \uparrow \infty}(v(z), w(z))=\left(v_{-}, 0\right), \\
v^{\prime}=w, \quad w^{\prime}=-s w-G_{-}(v) \text { for } z \notin[-m, m], \\
v^{\prime}=w, \quad w^{\prime}=-s w-G_{+}(v) \text { for } z \in(-m, m) .
\end{gathered}
$$

The system (2.3) has a unique equilibrium point $\left(v_{-}, 0\right)$. Since $G_{-}{ }^{\prime}<0$ and $s \geq 0$, the saddle point $\left(v_{-}, 0\right)$ has an unstable manifold $\mathbf{U}_{-}$and a stable manifold $\mathbf{S}_{-}$lying in the half plane $\left\{(v, w) ; v>v_{-}\right\}$. They are expressed as

$$
\mathbf{U}_{-}=\left\{(v, w) ; v>v_{-}, w=U_{-}(v ; s)\right\} \text { and } \mathbf{S}_{-}=\left\{(v, w) ; v>v_{-}, w=S_{-}(v ; s)\right\}
$$

respectively, by using smooth functions $U_{-}(v ; s)$ and $S_{-}(v ; s)$. Similarly, $\left(v_{+}, 0\right)$ is a unique equilibrium point of $(2.4)$, and $\left(v_{+}, 0\right)$ has a stable manifold $\mathbf{S}_{+}$and an unstable manifold $\mathbf{U}_{+}$lying in the half plane $\left\{(v, w) ; v<v_{+}\right\}$. We denote the unique cross point of $\mathbf{U}_{-}$and $\mathbf{S}_{+}$by $\left(\tilde{v}_{+}, \tilde{w}_{+}\right)$and that of $\mathbf{S}_{-}$and $\mathbf{U}_{+}$by $\left(\tilde{v}_{-}, \tilde{w}_{-}\right)$, respectively. Solution orbits $\mathbf{F}_{+}$of $(2.4)$ crossing the $v$-axis in the part of $v<v_{+}$are expressed as

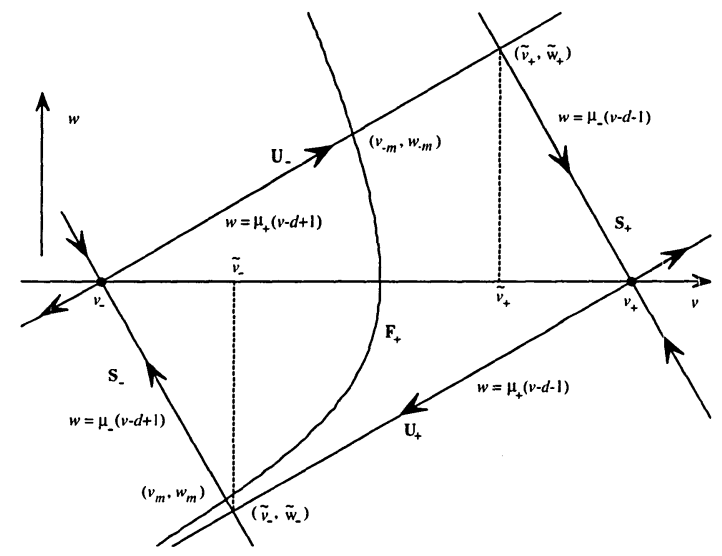

FIG. 2.1. $\mathbf{U}_{-}, \mathbf{S}_{-}, \mathbf{U}_{+}, \mathbf{S}_{+}, \mathbf{F}_{+}$and others

$$
F_{+}(v, w ; s)=\text { constant }
$$

by the use of a smooth function $F_{+}(v, w ; s)$. See Figure 2.1 , in which $\mathbf{U}_{-}, \mathbf{S}_{-}, \mathbf{U}_{+}$, $\mathbf{S}_{+}, \mathbf{F}_{+}$and others are depicted for the case of the nonlinearity (1.3).

We put $\left(v_{-m}, w_{-m}\right)=(v(-m), w(-m))$ and $\left(v_{m}, w_{m}\right)=(v(m), w(m))$. Since $c(v)$ is strictly monotone decreasing, the first two equations in (2.1) are rewritten as

$$
v_{-m}=c^{-1}(-\tau s) \text { and } v_{m}=c^{-1}(\tau s)
$$

by the use of the inverse function $c^{-1}$ of $c$. It follows from $(2.2) \sim(2.4)$ that

$$
\begin{gathered}
w_{-m}=U_{-}\left(v_{-m} ; s\right), \quad v_{-}<v_{-m}<\tilde{v}_{+}, \quad w_{m}=S_{-}\left(v_{m} ; s\right), \quad v_{-}<v_{m}<\tilde{v}_{-} \\
F_{+}\left(v_{-m}, w_{-m} ; s\right)=F_{+}\left(v_{m}, w_{m} ; s\right) .
\end{gathered}
$$


Substituting (2.5) and (2.6) into (2.7), we obtain

$$
\begin{gathered}
F_{+}\left(c^{-1}(-\tau s), U_{-}\left(c^{-1}(-\tau s) ; s\right) ; s\right)=F_{+}\left(c^{-1}(\tau s), S_{-}\left(c^{-1}(\tau s) ; s\right) ; s\right) \\
v_{-}<c^{-1}(-\tau s)<\tilde{v}_{+}, \quad v_{-}<c^{-1}(\tau s)<\tilde{v}_{-} .
\end{gathered}
$$

Thus, the problem of finding a travelling pulse of (1.4) is reduced to the problem of finding a solution $\{s \geq 0, \tau>0\}$ of (2.8).

3. Travelling front and back waves and standing pulses. Hereafter, we discuss the behavior of pulse solutions by adopting the piecewise-linear nonlinearity (1.3) as a prototype. The system (RD) becomes bistable when $p>1$ and $|d|<p-1$ in (1.3). If $p \geq 2$, the bistable condition $|d|<p-1$ is satisfied by all travelling pulses of (SL) constructed by fixing $\tau$ and adopting $d$ as a parameter in Section 4 and they can be extended to travelling pulses of (RD) with small $\epsilon>0$. If $1<p<2$, on the other hand, some travelling pulses of (SL) given in Section 4 violate the bistable condition (Proposition 4). This is the reason why we restrict $p \geq 2$ in (1.3). We may assume $d \geq 0$ without loss of generality since the relations

$$
f(-u,-v)=-f(u, v) \text { and } g(-u,-v ;-d)=-g(u, v ; d)
$$

imply that if $(u(t, x), v(t, x))$ is a solution of (RD) with $d=d^{*}$ then so is $(-u(t, x),-v(t, x))$ of (RD) with $d=-d^{*}$.

We briefly review the existence and stability of travelling front/back waves and standing pulses of the reaction-diffusion system (RD). All the propositions in this section can be extended to the reaction-diffusion systems (RD) with small $\epsilon>0$ although they are stated for the singular limit system (SL) for the brevity and the clearness. Travelling wave solutions $(u(t, x), v(t, x))$ of $(1.2)$ having exactly one transition layer are named travelling front waves and travelling back waves in [8]. A travelling front wave and a travelling back wave satisfy

$$
\lim _{x \rightarrow \pm \infty}(u(t, x), v(t, x))=\left(u_{\mp}, v_{\mp}\right) \text { and } \lim _{x \rightarrow \pm \infty}(u(t, x), v(t, x))=\left(u_{ \pm}, v_{ \pm}\right),
$$

respectively. Clearly a travelling front wave with velocity $s$ becomes a travelling back wave with velocity $-s$ through the coordinate transformation $x \rightarrow-x$. The stability of travelling front and back waves has been studied in [7], [11] and [20] for instance. Let us here apply the results to (SL). We fix $\tau>0$, and define

$$
v_{\tau}(S)=p \tau S\left(\tau^{2} S^{2}+1-S^{2}\right)^{-1 / 2} \text { and } d_{f}(S ; \tau)=S-v_{\tau}(S) \text { for } S \in(-1,1) .
$$

The smooth odd function $d_{f}(S ; \tau)$ satisfies

$$
\begin{gathered}
d_{f}(0 ; \tau)=0, \quad \lim _{S \rightarrow \pm 1} d_{f}(S ; \tau)= \pm(1-p) \\
d_{f}(S ; \tau)<0 \text { on }(0,1) \text { when } \tau \geq 1 / p, \\
d_{f}(S ; \tau)>0 \text { on }\left(0, S_{*}(\tau)\right) \text { and } d_{f}(S ; \tau)<0 \text { on }\left(S_{*}(\tau), 1\right) \text { when } \tau<1 / p,
\end{gathered}
$$

where $S_{*}(\tau)=\left\{\left(1-p^{2} \tau^{2}\right) /\left(1-\tau^{2}\right)\right\}^{1 / 2}$. By applying the discussion in [7] and [11], it is shown that for each $S \in(-1,1)$, (SL) has a travelling front wave with the velocity $s=2 S\left(1-S^{2}\right)^{-1 / 2}$ being unique up to phase shifts if and only if $d=d_{f}(S ; \tau)$ and the wave is stable if $d_{f}{ }^{\prime}(S ; \tau)<0$ while it is unstable if $d_{f}^{\prime}(S ; \tau)>0$.

For any $\tau>0$ travelling front and back waves with the same velocity 0 coexist at $d=0$. Similarly, when $\tau<1 / p$, stable travelling front and back waves with the 
same velocity $S_{*}(\tau)$ also coexist at $d=0$. The bifurcation of travelling pulses from these coexisting travelling front and back waves has been already proved (Fig. 11 of [8], Fig. 4 of [9] or Fig. 4.2 of [14]). We note that travelling pulses constructed in the present paper are bifurcated from these travelling front and back waves $((3.2)$ in Proposition 1 and (4.9) in Proposition 4).

Proposition 1. (1) Fix $\tau<1 / p$. There exist a positive number $\delta(\tau)<S_{*}(\tau)$ and a smooth function $d_{p}(S ; \tau)$ such that for each $S \in\left(S_{*}(\tau)-\delta(\tau), S_{*}(\tau)\right)(S L)$ has a stable travelling pulse with the velocity $s=2 S\left(1-S^{2}\right)^{-1 / 2}$ if and only if $d=d_{p}(S ; \tau)$. Moreover,

$$
\lim _{S \uparrow S_{*}(\tau)} d_{p}(S ; \tau)=0 \quad \text { and } \quad \lim _{S \uparrow S_{*}(\tau)} d_{p}{ }^{\prime}(S ; \tau)=\lim _{S \uparrow S_{*}(\tau)} d_{f}{ }^{\prime}(S ; \tau)=1-(p \tau)^{-2} .
$$

(2) Let $\tau \neq 1 / p$. Then, (SL) has a standing pulse for small d. Moreover, the standing pulse is stable if $\tau>1 / p$ while it has three unstable eigenvalues if $\tau<1 / p$.

H. Ikeda and T. Ikeda [10] have dealt with standing pulses of (1.2), and have shown that there exist two types of destabilization of standing pulses as $\tau$ decreases. One is the static bifurcation and the other is the Hopf bifurcation. Since the order of these two bifurcation points is apparently crucial for the pattern selection problem, they have also studied which type of bifurcation occurs first as $\tau$ decreases by using the nonlinearity (1.3) with $p=3 / 2$. Their results can be applied to (SL), which are summarized as

Proposition 2. (1) For $d \geq 1$ (SL) has no standing pulse. For each fixed $d \in(0,1)$ (SL) has a standing pulse, which is unique up to phase shifts. The width of its excited region is given by $-\log d$, which tends to $\infty$ and 0 as $d \downarrow 0$ and $d \uparrow 1$, respectively.

(2) There exist $\tau_{\text {static }}(d)>0$ and $\tau_{H}(d)>0$ such that the standing pulse is stable for $\tau>\max \left\{\tau_{\text {static }}(d), \tau_{H}(d)\right\}$, and it is destabilized through the static bifurcation at $\tau=\tau_{\text {static }}(d)$ and through the Hopf bifurcation at $\tau=\tau_{H}(d)$ as $\tau$ decreases.

(3) The smooth functions $\tau_{\text {static }}(d)$ and $\tau_{H}(d)$ satisfy

$$
\begin{aligned}
& \lim _{d \downarrow 0} \tau_{\text {static }}(d)=\lim _{d \downarrow 0} \tau_{H}(d)=1 / p, \quad \tau_{\text {static }}{ }^{\prime}(d)<0 \text { for any } d \in(0,1), \quad \lim _{d \downarrow 0} \tau_{H}{ }^{\prime}(d)>0, \\
& \tau_{\text {static }}(d)=O\left((1-d)^{2}\right) \quad \text { and } \quad \tau_{H}(d)=O\left((1-d)^{3}\right) \quad \text { around } d=1,
\end{aligned}
$$

and hence

$(3.3) \tau_{H}(d)>\tau_{\text {static }}(d)$ around $d=0$ while $\tau_{\text {static }}(d)>\tau_{H}(d)$ around $d=1$.

Each standing pulse of (SL) is symmetric with respect to the center of excited region. It is shown in [10] that the eigenvalue problems with an eigenvalue $\lambda$ around the standing pulse for the asymmetric and symmetric perturbations are expressed respectively by

$$
\begin{aligned}
& p \tau \lambda / 2-1+d+\left(1-e^{-2 m \kappa}\right) / \kappa=0 \text { and } \\
& p \tau \lambda / 2-1+d+\left(1+e^{-2 m \kappa}\right) / \kappa=0,
\end{aligned}
$$

where $\kappa=(1+\lambda)^{1 / 2}, \operatorname{Re} \kappa>0$, and $2 m=-\log d$ denotes the width of excited region. The equation (3.4) has two real solutions. One is the eigenvalue $\lambda_{0}=0$ corresponding 
to the translation of the standing pulse. (We often call this 0 -eigenvalue the trivial 0 eigenvalue in the present paper.) The other eigenvalue $\lambda_{1}$ changes its sign depending on $d$ and $\tau$. The static bifurcation point $\tau_{\text {static }}(d)$ is determined so that $\lambda_{1}=0$ and it is explicitly expressed as

$$
p \tau_{\text {static }}(d)-1+d-d \log d=0 .
$$

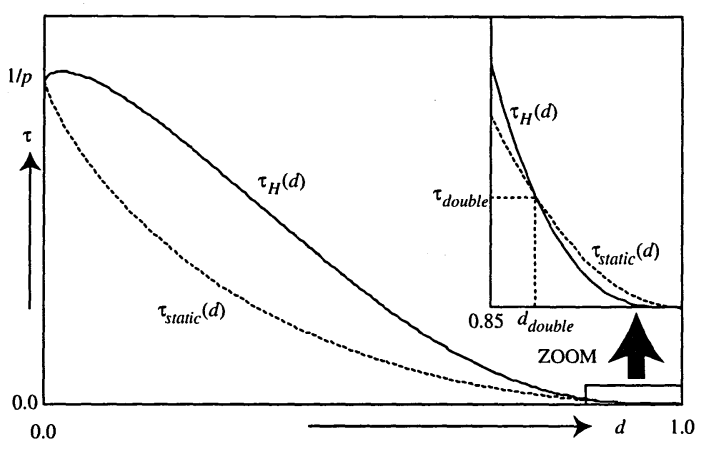

FIG. 3.1. The Hopf and static bifurcation points of standing pulses of (SL)

The equation (3.5) also has two solutions $\lambda_{2}$ and $\lambda_{3}$. Both $\lambda_{2}$ and $\lambda_{3}$ are negative real numbers for large $\tau$, however, they become a complex-conjugate-pair as $\tau$ decreases and cause the Hopf bifurcation at $\tau=\tau_{H}(d)$. The critical value $\tau_{H}(d)$ can be calculated by using the method (8) of Appendix, and Figure 3.1 shows the graphs of $\tau=\tau_{H}(d)$ and $\tau=\tau_{\text {static }}(d)$ with $p=2$. Thus, a standing pulse has four critical eigenvalues $\lambda_{0}, \lambda_{1}, \lambda_{2}$, and $\lambda_{3}$. The stability symbol of the standing pulse is $\{0,+,+,+\}$ for sufficiently small $d$, and it changes from $\{0,+,+,+\}$ to $\{0,+,+,-\}$ and $\{0,-,-,-\}$ in the order as $d$ increases if $\tau_{\text {static }}(d)<\tau_{H}(d)$ while the order is replaced by $\{0,+,+,+\},\{0,+,-,-\}$ and $\{0,-,-,-\}$ if $\tau_{H}(d)<\tau_{\text {static }}(d)$.

Although the bifurcation structure of standing pulses will be studied later in Section 6 for the case where $\tau$ is fixed, we get the following partial results at the present point:

- Let $\tau>\tau_{\text {peak }} \equiv \max _{d \in(0,1)} \tau_{H}(d)$. The standing pulse is stable for any $d \in(0,1)$.

- Let $\tau \in\left(1 / p, \tau_{\text {peak }}\right)$. The stable standing pulse for sufficiently small $d$ loses and recovers its stability through the Hopf bifurcation as $d$ increases.

- Let $\tau<1 / p$. The unstable standing pulse with stability symbol $\{0,+,+,+\}$ for sufficiently small $d$ recovers its stability through exactly one time static bifurcation as $d$ increases. It also recovers, loses and recovers the stability via the Hopf bifurcation as $d$ increases. Finally it becomes stable for large $d$.

4. Velocity of travelling pulses. This section is devoted to the study of the existence of travelling pulses of (SL). The functions $c(v), G_{-}(v)$ and $G_{+}(v)$ in (SL) are given by

$$
\begin{gathered}
c(v)=c(v ; p)=-2 v\left(p^{2}-v^{2}\right)^{-1 / 2} \\
G_{-}(v)=G_{-}(v ; d)=-v+d-1 \text { and } G_{+}(v)=G_{+}(v ; d)=-v+d+1
\end{gathered}
$$


The saddle points of (2.3) and (2.4) are given by $\left(v_{-}, 0\right)=(d-1,0)$ and $\left(v_{+}, 0\right)=$ $(d+1,0)$, respectively. We deal with travelling pulses having a localized excited region $(d>0)$ and a positive velocity $(s>0)$. Instead of the velocity $s>0$, a more convenient notation

$$
S=s\left(s^{2}+4\right)^{-1 / 2} \in(0,1)
$$

will be utilized hereafter. Then, (2.5) is replaced by

$$
v_{-m}=-v_{m}=v_{\tau}(S)
$$

and $U_{-}, S_{-}$and $F_{+}$in $(2.6) \sim(2.8)$ are expressed as

$$
\begin{gathered}
U_{-}(v ; S)=\mu_{+}(S)(v-d+1), \quad S_{-}(v ; S)=\mu_{-}(S)(v-d+1), \\
F_{+}(v, w ; S)=\left\{\mu_{-}(S)(v-d-1)-w\right\}^{1+S}\left\{w-\mu_{+}(S)(v-d-1)\right\}^{1-S}
\end{gathered}
$$

where

$$
\mu_{+}(S)=(1-S)\left(1-S^{2}\right)^{-1 / 2}>0 \text { and } \mu_{-}(S)=-(1+S)\left(1-S^{2}\right)^{-1 / 2}<0
$$

are solutions of the characteristic equation $x^{2}+s x-1=0$ of (2.3) and (2.4). Moreover, $\tilde{v}_{+}$and $\tilde{v}_{-}$in (2.8) are given by $\tilde{v}_{+}=d+S$ and $\tilde{v}_{-}=d-S$, respectively (Figure 2.1). Now, our problem of finding a travelling pulse of (SL) is to

Find $d>0, \tau>0$ and $0<S<1$ such that

$$
\begin{aligned}
(4.6) F\left(S, d, v_{\tau}(S)\right) & \equiv(1+S) \log \frac{d+S-v_{\tau}(S)}{1+S}-(1-S) \log \frac{d-S+v_{\tau}(S)}{1-S}=0 \\
d-1<v_{\tau}(S) & <d+S, \quad d-1<-v_{\tau}(S)<d-S
\end{aligned}
$$

since the first equality in (2.8) is equivalent to $F\left(S, d, v_{\tau}(S)\right)=0$. If $\{v(z), m ; s>0\}$ is a solution of (2.1) satisfying $\lim _{|z| \uparrow \infty} v(z)=v_{-}$then $\left\{d, \tau, S=s\left(s^{2}+4\right)^{-1 / 2}\right\}$ is a solution of (4.6) and $v(-m)=v_{\tau}(S)$, while if $\{d, \tau, S\}$ is a solution of (4.6) then one can find a unique function $v(z)$ and a positive number $m$ so that $\{v(z), m ; s=2 S(1-$ $\left.\left.S^{2}\right)^{-1 / 2}\right\}$ is a solution of (2.1) subject to the boundary condition $\lim _{|z| \uparrow \infty} v(z)=v_{-}$.

We have the following necessary condition for a solution of (4.6):

Proposition 3. A solution $\{d, \tau, S\}$ of (4.6) satisfies

$$
0<d<1-v_{\tau}(S) / S, \quad 0<\tau<1 / p \text { and } 0<S<S_{*}(\tau) \equiv\left\{\frac{1-p^{2} \tau^{2}}{1-\tau^{2}}\right\}^{1 / 2}
$$

We study the problem (4.6) first by fixing $\tau \in(0,1 / p)$. The following proposition implies that there exists a unique branch $\left\{\left(d_{\tau}(S), S\right) ; 0<S<S_{*}(\tau)\right\}$ of travelling pulses of (SL) in the $(d, S)$-plane for an arbitrarily fixed $\tau \in(0,1 / p)$, and that the branch connects the bifurcation point on the $S$-axis from travelling front/back waves and the static bifurcation point on the $d$-axis from standing pulses. It also clarifies the direction of the bifurcation of travelling pulse from standing pulses.

Proposition 4. Fix $\tau \in(0,1 / p)$. For each $S \in\left(0, S_{*}(\tau)\right)$ there exists a unique $d_{\tau}=d_{\tau}(S)$ such that $\left\{d_{\tau}, \tau, S\right\}$ is a solution of (4.6). Moreover, $d_{\tau}(S)$ satisfies

$$
S-v_{\tau}(S)<d_{\tau}(S)<1-v_{\tau}(S) / S,
$$




$$
\begin{aligned}
& \lim _{S \uparrow S_{*}(\tau)} d_{\tau}(S)=0 \quad \text { and } \quad \lim _{S \uparrow S_{*}(\tau)} d_{\tau}{ }^{\prime}(S)=1-(p \tau)^{-2}=\lim _{S \uparrow S_{*}(\tau)} d_{f}{ }^{\prime}(S ; \tau), \\
& p \tau-1+d_{\tau}(0)-d_{\tau}(0) \log d_{\tau}(0)=0 \quad \text { and } \quad d_{\tau}{ }^{\prime}(0)=0, \\
& \left(-3 \log d_{\tau}(0)\right) d_{\tau}^{\prime \prime}(0)= \\
& d_{\tau}(0)-d_{\tau}(0)\left(1-\log d_{\tau}(0)\right)^{2}\left(1+2 \log d_{\tau}(0)\right)-3 p \tau\left(1-\tau^{2}\right), \\
& d_{\tau}^{\prime \prime}(0)<0 \text { if } \tau>\tau_{c} \quad \text { while } \quad d_{\tau}^{\prime \prime}(0)>0 \text { if } \tau<\tau_{c},
\end{aligned}
$$

where $d_{\tau}(0)=\lim _{S \downarrow 0} d_{\tau}(S), d_{\tau}{ }^{\prime}(0)=\lim _{S \downarrow 0} d_{\tau}{ }^{\prime}(S), d_{\tau}{ }^{\prime \prime}(0)=\lim _{S \downarrow 0} d_{\tau}{ }^{\prime \prime}(S)$ and $\tau_{c}$ is some constant belonging to $(0,1 / p)$.

The reaction-diffusion system (RD) becomes bistable if and only if $|d|<p-1$. When $p \geq 2, d_{\tau}(S)$ always satisfies the bistable condition $|d|<p-1$ by (4.8). If $p<2$, on the other hand, the bistable condition is violated. Actually, we have

$$
p \tau=1-d_{\tau}(0)+d_{\tau}(0) \log d_{\tau}(0) \text { in the limit of } S \downarrow 0,
$$

which implies that $d_{\tau}(S)$ tends to 1 as $(\tau, S) \downarrow(0,0)$ and $d_{\tau}(S)>p-1$ for small $\tau$ and $S$.

We study (4.6) also by fixing $d \in(0,1)$, and obtain the following results similar to the case where $\tau \in(0,1 / p)$ is fixed: the unique existence of branch $\left\{\left(\tau_{d}(S), S\right) ; 0<\right.$ $S<1\}$ of travelling pulses in the $(\tau, S)$-plane, the connection by the branch between the bifurcation point on the $S$-axis from travelling front/back waves and the static bifurcation point on the $\tau$-axis from standing pulses, and the direction of static bifurcation from standing pulses.

Proposition 5. Fix $d \in(0,1)$. For each $S \in(0,1)$ there exists a unique $\tau_{d}=$ $\tau_{d}(S)$ such that $\left\{d, \tau_{d}(S), S\right\}$ is a solution of (4.6). Moreover, it holds that

$$
\begin{aligned}
& \lim _{S \uparrow 1} v_{\tau_{d}(S)}(S)=1-d \quad \text { and } \quad \lim _{S \uparrow 1} \tau_{d}(S)=0, \\
& \lim _{S \uparrow 1} \frac{d}{d S} v_{\tau_{d}(S)}(S)=1 \quad \text { and } \quad \lim _{S \uparrow 1} \frac{d}{d S}\left(\tau_{d}(S) \sqrt{1-S^{2}}\right)=\frac{-2(1-d)}{\left(p^{2}-(1-d)^{2}\right)^{1 / 2}}, \\
& \left\{\begin{array}{l}
p \tau_{d}(0)-1+d-d \log d=0, \quad \tau_{d}{ }^{\prime}(0)=0, \\
3 p \tau_{d}^{\prime \prime}(0)=d-d(1-\log d)^{2}(1+2 \log d)-3 p \tau_{d}(0)\left(1-\tau_{d}(0)^{2}\right) \quad \text { and } \\
\tau_{d}^{\prime \prime}(0)<0 \text { if } d<d_{c} \quad \text { while } \tau_{d}^{\prime \prime}(0)>0 \text { if } d>d_{c},
\end{array}\right.
\end{aligned}
$$

where $\tau_{d}(0)=\lim _{S \downarrow 0} \tau_{d}(S), \tau_{d}{ }^{\prime}(0)=\lim _{S \downarrow 0} \tau_{d}{ }^{\prime}(S), \tau_{d}^{\prime \prime}(0)=\lim _{S \downarrow 0} \tau_{d}^{\prime \prime}(S)$ and $d_{c} \in$ $(0,1)$ is the solution of $1-x+x \log x=p \tau_{c}$.

The above two propositions assure the existence of the global branch of travelling pulses and show that the branch has at least one turning point when $\tau$ is fixed in the interval $\left(0, \tau_{c}\right)$ or when $d$ is fixed in $\left(d_{c}, 1\right)$. The critical value $\tau_{c}$ is very near to $1 / p$ and $d_{c}$ is very near to 0 as shown in Figure 4.1. Actually $\tau_{c} \simeq 0.4793$ and $d_{c} \simeq 0.00692$ when $p=2$.

It is still not easy to check the sign of $d_{\tau}{ }^{\prime}(S)$ and $\tau_{d}{ }^{\prime}(S)$ for $S \in(0,1)$ in the rigorous way even for the piecewise-linear nonlinearity (1.3). We will give a conjecture on this problem derived from numerical computation of (4.6) in Section 6. 


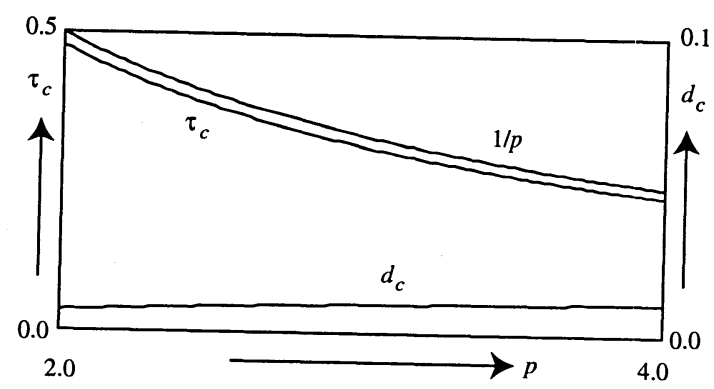

FIG. 4.1. Direction of static bifurcation

5. Stability of travelling pulses. Let $\{V(z), m ; s\}$ be a travelling pulse of (1.4), where $z=x-s t$ is the moving coordinate. We derive the formal linearized eigenvalue problem of (1.4) around $\{V(z), m ; s\}$. Substituting

$$
\begin{aligned}
& \psi_{-}(t)=s t-m-\phi_{-}(t), \quad \psi_{+}(t)=s t+m+\phi_{+}(t), \\
& v(t, x)=V(x-s t)+w(t, x-s t)=V(z)+w(t, z)
\end{aligned}
$$

into (1.4), where $s t-m$ and $s t+m$ denote respectively the left and right interface positions of the travelling pulse $V(z)$ and $\left\{w(t, z), \phi_{-}(t), \phi_{+}(t)\right\}$ is a perturbation, we
obtain

$$
\begin{aligned}
& \tau \frac{d \phi_{-}}{d t}(t)=\dot{c}(V(-m))\left\{-V^{\prime}(-m) \phi_{-}(t)+w(t,-m)\right\}+\text { h.o.t., } \\
& \tau \frac{d \phi_{+}}{d t}(t)=\dot{c}(V(m))\left\{V^{\prime}(m) \phi_{+}(t)+w(t, m)\right\}+\text { h.o.t. } \\
& \left(w_{t}, \xi\right)+\left(w_{x}, \xi^{\prime}\right)-\left(s w_{x}, \xi\right)=[G]_{V(-m)} \xi(-m) \phi_{-}(t)+[G]_{V(m)} \xi(m) \phi_{+}(t) \\
& \quad+\int_{-\infty}^{-m} \dot{G}_{-}(V) w \xi d z+\int_{m}^{\infty} \dot{G}_{-}(V) w \xi d z+\int_{-m}^{m} \dot{G}_{+}(V) w \xi d z+\text { h.o.t. }
\end{aligned}
$$

for any $\xi \in H^{1}(\mathbf{R})$.

Here $(, \quad)$ denotes the usual $L^{2}$-inner product on $\mathbf{R}$,

$$
\begin{aligned}
& \dot{c}(v)=\frac{d c}{d v}(v), \quad '=\frac{d}{d z}, \\
& \dot{G}_{ \pm}(V(z))=\frac{d G_{ \pm}}{d v}(V(z)) \text { and }[G]_{V(z)}=G_{+}(V(z))-G_{-}(V(z)) .
\end{aligned}
$$

Thus, the eigenvalue problem is given by

Find $\lambda \in \mathbf{C}, \phi_{-} \in \mathbf{C}, \phi_{+} \in \mathbf{C}$, and $w \in H^{1}(\mathbf{R})$ such that

$$
\begin{aligned}
& \tau \lambda \phi_{-}=\dot{c}(V(-m))\left\{-V^{\prime}(-m) \phi_{-}+w(-m)\right\} \\
& \tau \lambda \phi_{+}=\dot{c}(V(m))\left\{V^{\prime}(m) \phi_{+}+w(m)\right\} \\
& \lambda(w, \xi)+\left(w^{\prime}, \xi^{\prime}\right)-\left(s w^{\prime}, \xi\right)=[G]_{V(-m)} \xi(-m) \phi_{-}+[G]_{V(m)} \xi(m) \phi_{+} \\
& \quad+\int_{-\infty}^{-m} \dot{G}_{-}(V) w \xi d z+\int_{m}^{\infty} \dot{G}_{-}(V) w \xi d z+\int_{-m}^{m} \dot{G}_{+}(V) w \xi d z
\end{aligned}
$$

for any $\xi \in H^{1}(\mathbf{R})$. 
Clearly, $\left\{\lambda=0, \phi_{-}=1, \phi_{+}=-1, w=V^{\prime}\right\}$ is a solution of (5.2) corresponding to the translation free of the travelling pulse $V(z)$.

We study the behavior of spectra of (5.2) with the nonlinearity (1.3). We have

$$
\begin{aligned}
& V(-m)=-V(m)=v_{\tau}(S) \\
& V^{\prime}(-m)=(1-S)\left(1-S^{2}\right)^{-1 / 2}\left(v_{\tau}(S)-d+1\right), \\
& V^{\prime}(m)=-(1+S)\left(1-S^{2}\right)^{-1 / 2}\left(-v_{\tau}(S)-d+1\right), \\
& \dot{c}(V(-m))=\dot{c}(V(m))=(-2 / p)\left(\tau^{2} S^{2}+1-S^{2}\right)^{3 / 2}\left(1-S^{2}\right)^{-3 / 2}, \\
& \dot{G}_{-}(V) \equiv \dot{G}_{+}(V) \equiv-1 \text { and }[G]_{V(-m)} \equiv[G]_{V(m)} \equiv 2,
\end{aligned}
$$

where $S=s\left(s^{2}+4\right)^{-1 / 2}$ and $v_{\tau}(S)$ is given by (4.4). Hence, (5.2) becomes

Find $\lambda \in \mathbf{C}, \phi_{-} \in \mathbf{C}, \phi_{+} \in \mathbf{C}$, and $w \in H^{1}(\mathbf{R})$ such that

$$
\begin{aligned}
& \hat{\tau} \lambda \phi_{-}=V^{\prime}(-m) \phi_{-}-w(-m), \quad \hat{\tau} \lambda \phi_{+}=-V^{\prime}(m) \phi_{+}-w(m), \\
& \lambda(w, \xi)+\left(w^{\prime}, \xi^{\prime}\right)-\left(s w^{\prime}, \xi\right)+(w, \xi)=2 \xi(-m) \phi_{-}+2 \xi(m) \phi_{+} \text {for any } \xi \in H^{1}(\mathbf{R}),
\end{aligned}
$$

where $\hat{\tau}=-\tau / \dot{c}(V(-m))$. The third equation of (5.4) is equivalent to

$$
\begin{gathered}
w^{\prime \prime}+s w^{\prime}-w=\lambda w \quad(z \neq \pm m), \\
w^{\prime}(-m-0)-w^{\prime}(-m+0)=2 \phi_{-} \quad \text { and } \quad w^{\prime}(m-0)-w^{\prime}(m+0)=2 \phi_{+} .
\end{gathered}
$$

We consider spectra of (5.4) belonging to $\{\zeta \in \mathbf{C} ; \operatorname{Re} \zeta>-1\}$. Solving (5.5) with respect to $w$ and substituting it into the first two equalities in (5.4), we obtain

$$
\left\{\begin{array}{l}
\hat{\tau} \lambda \phi_{-}=V^{\prime}(-m) \phi_{-}-\frac{\phi_{-}+\phi_{+} e^{-2 m \kappa_{+}}}{\kappa^{2 m \kappa_{-}}} \\
\hat{\tau} \lambda \phi_{+}=-V^{\prime}(m) \phi_{+}-\frac{\phi_{+}+\phi_{-} e^{2 m \kappa}}{\kappa}
\end{array}\right.
$$

where $\kappa_{ \pm}=\kappa_{ \pm}(\lambda)$ are two solutions of the characteristic equation $x^{2}+s x-(1+\lambda)=0$ $\left(\operatorname{Re} \kappa_{-}<0 \leq \operatorname{Re} \kappa_{+}\right)$, and $\kappa=\kappa(\lambda)=\left(\kappa_{+}(\lambda)-\kappa_{-}(\lambda)\right) / 2$, that is,

$$
\begin{gathered}
\kappa_{ \pm}(\lambda)=-s / 2 \pm\left(s^{2} / 4+1+\lambda\right)^{1 / 2}, \quad \kappa_{ \pm}(0)=\mu_{ \pm}=(-S \pm 1)\left(1-S^{2}\right)^{-1 / 2} \\
\kappa(\lambda)=\left(s^{2} / 4+1+\lambda\right)^{1 / 2} \text { and } \kappa(0)=\left(1-S^{2}\right)^{-1 / 2}
\end{gathered}
$$

Of course, (5.6) has a non-trivial solution if and only if

$$
\left(\hat{\tau} \lambda-V^{\prime}(-m)+1 / \kappa\right)\left(\hat{\tau} \lambda+V^{\prime}(m)+1 / \kappa\right)-e^{-4 m \kappa} / \kappa^{2}=0,
$$

which is the equation determining eigenvalues of (5.4). Since $\left\{\lambda=0, \phi_{-}=1, \phi_{+}=\right.$ $-1\}$ is a solution of (5.6) corresponding to the translation of travelling pulse, we know

$$
V^{\prime}(-m)=\left(1-e^{-2 m \kappa_{+}(0)}\right) / \kappa(0) \text { and } V^{\prime}(m)=-\left(1-e^{2 m \kappa_{-}(0)}\right) / \kappa(0) .
$$

By the use of (5.3), (5.7) and (5.9), we have the expression of the width of excited region

$$
2 m=-\frac{\left(1-S^{2}\right)^{1 / 2}}{1-S} \log \frac{d+S-v_{\tau}(S)}{1+S}=-\frac{\left(1-S^{2}\right)^{1 / 2}}{1+S} \log \frac{d-S+v_{\tau}(S)}{1-S}
$$


Let us fix $\tau<1 / p$. The eigenvalue problem (5.8) is defined on the branch $\left\{\left(d_{\tau}(S), S\right) ; 0<S<S_{*}(\tau)\right\}$ in $(d, S)$-plane, and its solution is continuous on the branch. The eigenvalue problem around a standing pulse is given by

$$
\left\{p \tau \lambda / 2-1+d+\left(1-e^{-2 m \kappa}\right) / \kappa\right\}\left\{p \tau \lambda / 2-1+d+\left(1+e^{-2 m \kappa}\right) / \kappa\right\}=0
$$

from (3.4) and (3.5). The problem (5.11) is defined on the $d$-axis in $(d, S)$-plane, and its solution is continuous there. The following proposition clarifies the continuity of (5.8) and (5.11) at the static bifurcation point of standing pulses.

Proposition 6. When $\tau<1 / p$ is fixed, the solution of (5.8) tends to that of (5.11) with $d=d_{\tau}(0)$ as $S \downarrow 0$. Similarly when $d \in(0,1)$ is fixed, the solution of (5.8) tends to that of (5.11) with $\tau=\tau_{d}(0)$ as $S \downarrow 0$.

For studying the behavior of real eigenvalue $\rho$ of (5.8), we put

$$
\begin{gathered}
H_{-}(\rho)=\hat{\tau} \rho-V^{\prime}(-m)+1 / \kappa(\rho), \quad H_{+}(\rho)=\hat{\tau} \rho+V^{\prime}(m)+1 / \kappa(\rho), \\
H_{0}(\rho)=e^{-4 m \kappa(\rho)} / \kappa(\rho)^{2} \text { and } H(\rho)=H_{-}(\rho) H_{+}(\rho)-H_{0}(\rho) .
\end{gathered}
$$

Then, (5.8) is equivalent to $H(\rho)=0$, and we have

Proposition 7. Put $\dot{H}(\rho)=\frac{d H}{d \rho}(\rho)$. It holds that

$$
\operatorname{sign} \dot{H}(0)=-\operatorname{signd}_{\tau}{ }^{\prime}(S)=-\operatorname{sign}_{d}{ }^{\prime}(S) \text {. }
$$

A close relation is expected between the sign of $\dot{H}(0)$ and the existence of positive eigenvalue of (5.8). It is easy to show that (5.8) has a positive eigenvalue if $\dot{H}(0)<0$ since $\lim _{\rho \uparrow \infty} H(\rho)=\infty$. We have also get the following partial result in the case of $\dot{H}(0)>0$ :

Proposition 8. (1) If $d_{\tau}{ }^{\prime}(S)>0$ (equivalently if $\tau_{d}{ }^{\prime}(S)>0$ ), then (5.8) has a positive eigenvalue and the travelling pulse is unstable.

(2) (5.8) has no non-negative real eigenvalue except the trivial 0-eigenvalue corresponding to the translation free of the travelling pulse when $0<\tau<1 / p$ is fixed and $S$ is near $S_{*}(\tau)$ or when $0<d<1$ is fixed and $S$ is near 1 .

In order to study the sign of real eigenvalues in the case of $\dot{H}(0)>0$ and to track the behavior of complex eigenvalues in accordance with $S$, we have to solve (5.8) and (5.11) numerically. The numerical method and the results will be discussed in Section 6 as well as a global bifurcation picture of standing and travelling pulses.

6. Global bifurcation diagram of pulse solutions. The static bifurcation point $\tau_{\text {static }}(d)$ of a standing pulse of (SL) is a monotone decreasing function explicitly given by (3.6). Although we do not have an explicit expression of the Hopf bifurcation point $\tau_{H}(d)$, we can calculate $\tau_{H}(d)$ with very high accuracy by using the method described in (8) of Appendix. Their graphs shown in Figure 3.1 lead us to the conjecture that there exist constants $d_{\text {peak }}$ and $d_{\text {double }}$ in the interval $(0,1)$ such that

$$
\begin{array}{rll}
\tau_{H}{ }^{\prime}(d)>0 \text { on }\left(0, d_{\text {peak }}\right) & \text { and } & \tau_{H}{ }^{\prime}(d)<0 \text { on }\left(d_{\text {peak }}, 1\right) \\
\tau_{H}(d)>\tau_{\text {static }}(d) \text { on }\left(0, d_{\text {double }}\right) & \text { and } & \tau_{H}(d)<\tau_{\text {static }}(d) \text { on }\left(d_{\text {double }}, 1\right) .
\end{array}
$$


We thus arrive at the following conjecture, where $\tau_{\text {peak }}=\tau_{H}\left(d_{\text {peak }}\right), \tau_{\text {double }}=$ $\tau_{H}\left(d_{\text {double }}\right)=\tau_{\text {static }}\left(d_{\text {double }}\right)$, and $\tau_{H}{ }^{-1}$ and $\tau_{\text {static }}{ }^{-1}$ are the inverse functions of $\tau_{H}$ and $\tau_{\text {static }}$, respectively:

CONJECTURE 1. (1-1) If $\tau>\tau_{\text {peak }}$, the standing pulse is stable for any $d \in(0,1)$. (1-2) Fix $\tau \in\left(1 / p, \tau_{p e a k}\right)$. There exist $0<d_{1}<d_{2}<1$ such that as $d$ decreases the stable standing pulse loses and recovers its stability through the Hopf bifurcations at $d=d_{2}$ and $d=d_{1}$, respectively. Here $d_{1}=d_{1}(\tau)$ tends to 0 as $\tau \downarrow 1 / p$.

(1-3) Fix $\tau \in\left(\tau_{\text {double }}, 1 / p\right)$. As d decreases, the standing pulse loses its stability first through the Hopf bifurcation at $d=\tau_{H}{ }^{-1}(\tau)$ and second via the static bifurcation at $d=\tau_{\text {static }}{ }^{-1}(\tau)$.

(1-4) Fix $\tau<\tau_{\text {double. }}$. As d decreases, the standing pulse loses its stability first through the static bifurcation at $d=\tau_{\text {static }}{ }^{-1}(\tau)$ and second via the Hopf bifurcation at $d=$ $\tau_{H}{ }^{-1}(\tau)$.

(2-1) Fix $d \in\left(d_{\text {double }}, 1\right)$. As $\tau$ decreases, the standing pulse loses its stability first through the static bifurcation at $\tau=\tau_{\text {static }}(d)$ and second via the Hopf bifurcation at $\tau=\tau_{H}(d)$.

(2-2) Fix $d \in\left(0, d_{\text {double }}\right)$. As $\tau$ decreases, the standing pulse loses its stability first through the Hopf bifurcation at $\tau=\tau_{H}(d)$ and second via the static bifurcation at $\tau=\tau_{\text {static }}(d)$.

The critical number $d_{c}$ appearing in (4.15) is very small as shown in Figure 4.1, so that we may suppose $d_{c}<d_{\text {double }}$ (equivalently, $\tau_{c}>\tau_{\text {double }}$ ). Combining this property and Conjecture 1 , we obtain

CONJECTURE 2. A travelling pulse bifurcated from a standing pulse at the static bifurcation point is unstable.

The branches $\left\{\left(d_{\tau}(S), S\right) ; 0<S<S_{*}(\tau)\right\}$ in the $(d, S)$-plane and $\left\{\left(\tau_{d}(S), S\right) ; 0<\right.$ $S<1\}$ in the $(\tau, S)$-plane of travelling pulses can be numerically calculated from (4.6) with very high accuracy by using the method explained in (9) of Appendix. We have thus obtained the following precise information on the branches (Figure 6.1).

Conjecture 3. (1) If $\tau>\tau_{c}$ is fixed, then $d_{\tau}(S)$ decreases with increasing $S$ on $\left(0, S_{*}(\tau)\right)$. If $\tau<\tau_{c}$ is fixed, then the branch has exactly one turning point $Q_{\text {turn }}(\tau)$. (2) If $d<d_{c}$ is fixed, then $\tau_{d}(S)$ decreases with increasing $S$. If $d>d_{c}$ is fixed, then the branch $\left\{\left(\tau_{d}(S), S\right) ; 0<S<1\right\}$ has exactly one turning point $P_{\text {turn }}(d)$.
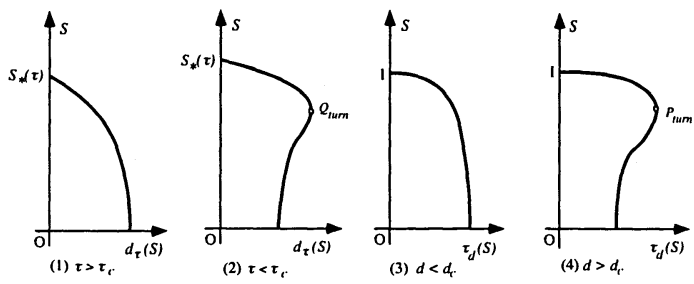

FIG. 6.1. Branches of travelling pulses ( $\tau$ is fixed in (1), (2) and $d$ is fixed in (3), (4))

When $\tau<1 / p$, stable travelling front and back waves with the same positive velocity coexist at $d=0$ as stated in Section 3. Both the front wave and the back wave have two critical eigenvalues: one is the 0-eigenvalue corresponding to the phase 
shifts and the other is negative. The travelling pulse bifurcated from the pair of coexisting travelling front and back waves inherits their critical eigenvalues. Actually it has four critical eigenvalues, one trivial 0-eigenvalue and three negative eigenvalues as proved in [8] and [9]. Each standing pulse also has four critical eigenvalues as stated in Section 3. These together with Propositions 6 lead us to the conjecture that (5.8) also has four critical eigenvalues. Based on this conjecture, we have calculated solutions of (5.8) by the high-accuracy method discussed in (10) of Appendix, and have obtained the following numerical evidence.
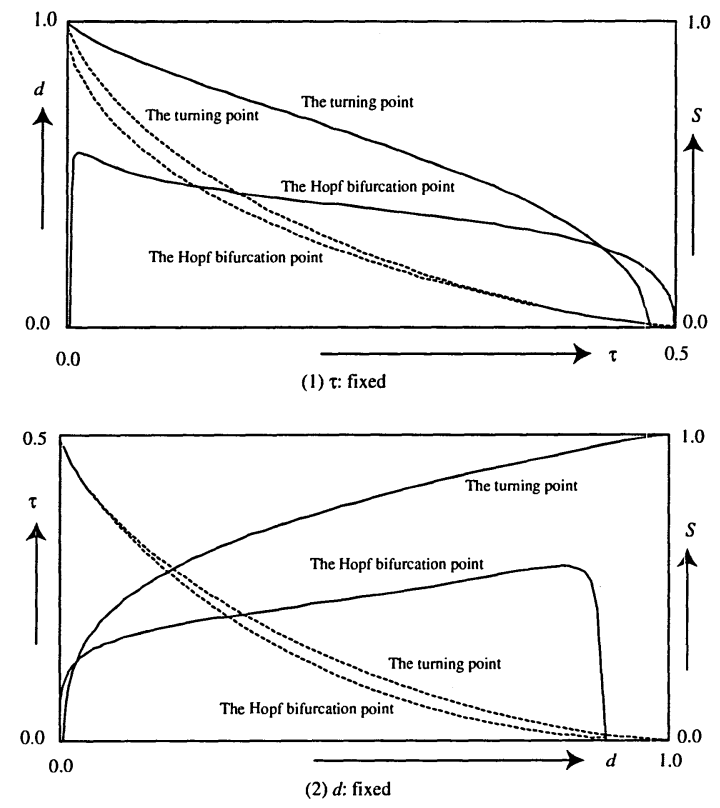

FIG. 6.2. The Hopf bifurcation point and turning point on the branch of travelling pulses $(p=2)$. The solid lines express the $S$-component $\left(S=s\left(s^{2}+4\right)^{-1 / 2}\right)$, and the dotted lines show the $d$-component in (1) and the $\tau$-component in (2), respectively.

Conjecture 4. (1) The branch $\left\{\left(d_{\tau}(S), S\right) ; 0<S<S_{*}(\tau)\right\}$ of travelling pulses contains no point where (5.8) has a non-trivial 0 -eigenvalue if $\tau>\tau_{c}$ while $Q_{\text {turn }}(\tau)$ is the unique point on the branch where (5.8) has a non-trivial 0-eigenvalue if $\tau<\tau_{c}$. The branch contains exactly one Hopf bifurcation point $Q_{\text {Hopf }}(\tau)$ if $\tau>\tau_{\text {double }}$ while it has no Hopf bifurcation point if $\tau<\tau_{\text {double }}$.

(2) The branch $\left\{\left(\tau_{d}(S), S\right) ; 0<S<1\right\}$ contains no point where (5.8) has a non-trivial 0 -eigenvalue if $d<d_{c}$ while $P_{\text {turn }}(d)$ is the unique point on the branch where (5.8) has a non-trivial 0-eigenvalue if $d>d_{c}$. The branch contains exactly one Hopf bifurcation point $P_{\text {Hopf }}(d)$ if $d<d_{\text {double }}$ while it has no Hopf bifurcation point if $d>d_{\text {double }}$.

The travelling pulse bifurcated from the coexisting travelling front and back waves is stable as proved in [8] and [9]. Hence, if the $S$-component of $Q_{H o p f}(\tau)$ is greater than that of $Q_{\text {turn }}(\tau)$, that is, if the Hopf bifurcation first occurs when $d$ increases, there is a possibility that a stable travelling breather bifurcates from the travelling pulse. If the order is reversed, on the other hand, no stable solution can arise from the Hopf bifurcation point. In this sense, the estimate of the order of $S$-components of $Q_{\text {Hopf }}(\tau)$ and $Q_{t u r n}(\tau)$ is crucial for the justification of stable travelling breather. 
Figure 6.2 compares $S$-components of $Q_{\text {Hopf }}(\tau)$ and $Q_{t u r n}(\tau)$ and those of $P_{H o p f}(d)$ and $P_{\text {turn }}(d)$, which are numerically calculated for $p=1 / 2$ by the method stated in (10) of Appendix. We thus arrive at the following conjecture.

CONJECTURE 5. (1) If $\tau<1 / p$ is fixed near $1 / p$, then a stable travelling breather bifurcates from the travelling pulse at $Q_{\text {Hopf }}(\tau)$ when $d$ increases.

(2) If $d \in(0,1)$ is fixed near 0 , then a stable travelling breather bifurcates from the travelling pulse at $P_{\text {Hopf }}(d)$ when $\tau$ increases.

7. Concluding remarks. The bifurcation pictures of standing and travelling pulses of (RD) becomes clearer when we draw them by fixing $\tau>0$ and adopting $d$ as a bifurcation parameter. We here explain the conjecture of bifurcation pictures in the $(d, s)$-plane drawn in Figure 1.6.

Fix a sufficiently small $\epsilon>0$. There exists $\tau_{\text {org }}^{\epsilon}>0$ such that (RD) has no travelling pulse with non-zero velocity if $\tau>\tau_{\text {org }}^{\epsilon}$ is fixed while (RD) has a unique branch $\left\{\left(d_{\tau}^{\epsilon}(s), s\right) ; 0<s<s_{*}\right\}$ of travelling pulses in the $(d, s)$-plane if $\tau<\tau_{o r g}^{\epsilon}$ is fixed ( $\lim _{\epsilon \downarrow 0} \tau_{o r g}^{\epsilon}=1 / p$ ). Moreover, the bifurcation picture of standing and travelling pulses changes as follows depending on the value of fixed $\tau>0$.

(1) All standing pulses are stable if $\tau>\tau_{\text {peak }}^{\epsilon} \equiv \max _{d \in(0,1)} \tau_{H}^{\epsilon}(d)$.

(2) There appears a pair of the Hopf bifurcation points $H_{-}^{\epsilon}$ and $H_{+}^{\epsilon}$ on the branch of standing pulses at $\tau=\tau_{\text {peak }}^{\epsilon}$, and $H_{-}^{\epsilon}$ tends to the origin while $H_{+}^{\epsilon}$ tends to $Q_{H}^{\epsilon}\left(\tau_{\text {org }}^{\epsilon}\right)$ as $\tau \downarrow \tau_{o r g}^{\epsilon}$. The case $\tau=\tau_{o r g}^{\epsilon}$ is the organizing center of the bifurcation diagram. Except $Q_{H}^{\epsilon}\left(\tau_{o r g}^{\epsilon}\right)$, all other critical points shrink up into the origin at $\tau=\tau_{\text {org }}^{\epsilon}$.

(3) When $\tau$ becomes below $\tau_{\text {org }}^{\epsilon}, Q_{\text {static }}^{\epsilon}(\tau)$ arises from the origin and a branch of travelling pulses appears also in a neighborhood of the origin. A travelling pulse bifurcates super-critically from $Q_{\text {static }}^{\epsilon}(\tau)$ and it recovers its stability at the Hopf bifurcation point $Q_{\text {Hopf }}^{\epsilon}(\tau)$ on the branch of travelling pulses.

(4) The direction of pitchfork bifurcation changes at $\tau=\tau_{c}^{\epsilon}$. The branch of travelling pulses contains a turning point $Q_{t u r n}^{\epsilon}(\tau)$ when $\tau<\tau_{c}^{\epsilon}$. The travelling pulse arising sub-critically from $Q_{\text {static }}^{\epsilon}(\tau)$ recovers the stability first at $Q_{\text {turn }}^{\epsilon}(\tau)$ and then at $Q_{\text {Hopf }}^{\epsilon}(\tau)$ for $\tau \in\left(\tau_{\text {Hopf }}^{\epsilon}, \tau_{c}^{\epsilon}\right)$, where $\tau_{\text {Hopf }}^{\epsilon}$ is the $\tau$-component of $P_{\text {Hopf }}^{\epsilon}\left(d_{\text {Hopf }}^{\epsilon}\right)$.

(5) The bifurcation diagram for $\tau \in\left(\tau_{\text {double }}^{\epsilon}, \tau_{\text {Hopf }}^{\epsilon}\right)$ is similar to the case (4) except the order between $Q_{\text {turn }}^{\epsilon}(\tau)$ and $Q_{\text {Hopf }}^{\epsilon}(\tau)$, where $\tau_{\text {double }}^{\epsilon}=\tau_{H}^{\epsilon}\left(d_{\text {double }}^{\epsilon}\right)$. As $\tau \downarrow \tau_{\text {double }}^{\epsilon}$, $Q_{\text {Hopf }}^{\epsilon}(\tau)$ approaches to the bifurcation point $Q_{\text {static }}^{\epsilon}\left(\tau_{\text {double }}^{\epsilon}\right) \equiv Q_{H}^{\epsilon}\left(\tau_{\text {double }}^{\epsilon}\right)$.

(6) The order between $Q_{H}^{\epsilon}(\tau)$ and $Q_{\text {static }}^{\epsilon}(\tau)$ is reversed at $\tau=\tau_{\text {double }}^{\epsilon}$. A travelling pulse arises sub-critically from $Q_{\text {static }}^{\epsilon}(\tau)$ and it recovers the stability at $Q_{\text {turn }}^{\epsilon}(\tau)$. Both $Q_{H}^{\epsilon}(\tau)$ and $Q_{\text {static }}^{\epsilon}(\tau)$ tend to $(1,0)$ as $\tau \downarrow 0$, and $\lim _{\tau \downarrow 0} Q_{\text {turn }}^{\epsilon}(\tau)=(1, \infty)$.

We studied the global bifurcation diagram of standing and travelling pulses of some bistable reaction-diffusion systems, and succeeded in showing the Hopf bifurcation of travelling pulses. We showed also that a stable travelling breather can come into view only when the nonlinearity is close to the odd symmetry. In order to complete the existence of unique branch of travelling pulses and in order to investigate the stability of travelling pulses along the branch, we assumed the piecewise-linear nonlinearity (1.3) and we sought the help of numerical computations of solutions of transcendental equations. Nevertheless, our results are expected to be valid to a wide class of nonlinearities satisfying the conditions (A1) (A4) since a variety of numerical evidences support the story of the advent of travelling breather from travelling pulses via the Hopf bifurcation, the typical example displayed in Figures 1.1 and 1.2 among others. 
As shown in Figure 1.6 a stable travelling breather bifurcates from $Q_{H o p f}^{\epsilon}$ when $\tau \in\left(\tau_{\text {Hopf }}^{\epsilon}, \tau_{\text {org }}^{\epsilon}\right)$ is fixed and $d$ is increased. What is the stability of travelling breather for much larger $d$ ? Aoki [1] has studied this problem by numerically solving the evolution systems and has shown that the travelling breather is destabilized by a saddle-node bifurcation when $d$ arrives at another critical value. The destabilization by saddle-node bifurcation is also proved for a layer-oscillating propagating pulse solution of an ODE model reduced from (1.4) in the forthcoming paper [17].

We close the present paper by noting the possibility of another origin of travelling breather. When $\tau=\tau_{\text {double }}^{\epsilon}$ is fixed and $d$ is decreased, the standing pulse loses its stability by the Hopf and static bifurcation at the same $d=d_{H}^{\epsilon}\left(\tau_{\text {double }}^{\epsilon}\right) \equiv d_{H}^{\epsilon}\left(\tau_{\text {double }}^{\epsilon}\right)$. That is, the bifurcation of codimension 2 occurs there. Complicated behaviors of solutions are expected around the codimension 2 bifurcation point in general. As reported in [1], rather simple and beautiful travelling breathers can appear near the point where both symmetric in-phase mode and asymmetric travelling mode bifurcate from the standing pulse. The codimension 2 bifurcation point is far away from the curve $\left\{Q_{\text {Hopf }}^{\epsilon}(\tau) ; \tau \in\left(\tau_{\text {Hopf }}^{\epsilon}, \tau_{o r g}^{\epsilon}\right)\right\}$ consisting of the Hopf bifurcation points of travelling pulses in the parameter space $\{(\tau, d) ; \tau>0, d \in(0,1)\}$. The connection by a sheet of travelling breathers between the codimension 2 bifurcation point and the curve $\left\{Q_{\text {Hopf }}^{\epsilon}(\tau)\right\}$ in the parameter space is carefully studied in [1] by numerically solving the evolution systems. At the present stage, however, the problem is still open and any numerical evidence is not yet proposed.

8. Appendix. This appendix states precise assumptions on the nonlinearity $\{f, g\}$, gives the proofs of propositions asserted in Sections 4 and 5 and describes the methods of numerical calculations referred in Section 6.

(1) Assumptions on the nonlinearity The results in Section 3 hold for a wider class of nonlinearities satisfying less restrictive assumptions, however, we impose the following conditions on $\{f, g\}$ throughout the present paper for the brevity.

(A1) The nullcline of $f$ is S-shaped and consists of three continuous curves $u=$ $h_{-}(v), u=h_{0}(v)$ and $u=h_{+}(v)$ defined on the intervals $\left(v_{\min }, \infty\right),\left(v_{\min }, v_{\max }\right)$ and $\left(-\infty, v_{\max }\right)$, respectively. The inequalities $h_{-}(v)<h_{0}(v)<h_{+}(v)$ hold on $\left(v_{\min }, v_{\max }\right)$.

(A2) $J(v) \equiv \int_{h_{-}(v)}^{h_{+}(v)} f(s, v) d s$ has an isolated zero at $v=v^{*} \in\left(v_{\min }, v_{\max }\right)$ and $\frac{d J}{d v}\left(v^{*}\right)<0$.

(A3) The nullcline of $g$ intersects with each of three parts of the nullcline of $f$ at $\left(u_{-}, v_{-}\right),\left(u_{0}, v_{0}\right)$ and $\left(u_{+}, v_{+}\right)$, respectively, and $v_{-}<v^{*}<v_{+}$.

(A4) $f_{v}(u, v)<0, g_{u}(u, v)>0$ and $g_{v}(u, v)<0$ on $\mathbf{R}^{2}, f_{u}\left(h_{-}(v), v\right)<0$ on $\left[v_{\text {min }}, \infty\right)$, $f_{u}\left(h_{+}(v), v\right)<0$ on $\left(-\infty, v_{\max }\right], \frac{d}{d v} g\left(h_{-}(v), v\right)<0$ on $\left[v_{\min }, \infty\right)$ and $\frac{d}{d v} g\left(h_{+}(v), v\right)<$ 0 on $\left(-\infty, v_{\max }\right]$.

(2) Proof of Proposition 3 Assume $\{d, \tau, S\}$ to be a solution of (4.6). The estimate $d<1$ follows immediately from $d-1<v_{\tau}(S)$ and $d-1<-v_{\tau}(S)$ in (4.6). The inequalities $d-1<v_{\tau}(S)<d+S$ and $d-1<-v_{\tau}(S)<d-S$ yield that

$$
0<\left(d+S-v_{\tau}(S)\right) /(1+S)<1 \text { and } 0<\left(d-S+v_{\tau}(S)\right) /(1-S)<1 \text {. }
$$

These together with $F\left(S, d, v_{\tau}(S)\right)=0$ lead to

$$
0<\left(d-S+v_{\tau}(S)\right) /(1-S)<\left(d+S-v_{\tau}(S)\right) /(1+S)<1,
$$


which implies $d<1-v_{\tau}(S) / S$. Thus, the proof is completed since $1-v_{\tau}(S) / S>0$ is equivalent to $\left(1-\tau^{2}\right) S^{2}<1-p^{2} \tau^{2}$.

(3) Proof of Proposition 4 The inequalities $0<\tau<1 / p$ and $0<S<S_{*}(\tau)$ lead to $S>v_{\tau}(S)>0$. The argument $d$ of $F\left(S, d, v_{\tau}(S)\right)$ can move only on the interval $\left(S-v_{\tau}(S), \eta(S)\right)$ by $(4.7)$, where $\eta(S)=1-v_{\tau}(S) / S$. It is clear that

$$
\begin{gathered}
\lim _{d \downarrow S-v_{\tau}(S)} F\left(S, d, v_{\tau}(S)\right)=+\infty, \\
F\left(S, \eta(S), v_{\tau}(S)\right)=2 S \log \eta(S)<0 .
\end{gathered}
$$

Now, the unique existence of solution and the property (4.8) follows from the facts that

$$
\frac{\partial F}{\partial d}\left(S, d, v_{\tau}\right)=\frac{1+S}{d+S-v_{\tau}(S)}-\frac{1-S}{d-S+v_{\tau}(S)}<0 \text { for } S-v_{\tau}(S)<d<\eta(S)
$$

The first property of (4.9) follows immediately from (4.8). Let us show the second property. Putting $d_{\tau}(S)=\delta(S) \eta(S)$ by $(4.8)$, where $\delta(S) \in(S, 1)$, and substituting this into $F\left(S, d(S), v_{\tau}(S)\right)=0$, we obtain

$$
(1+S) \log \frac{\delta(S)+S}{1+S}-(1-S) \log \frac{\delta(S)-S}{1-S}+2 S \log \eta(S)=0 .
$$

As $S$ tends to $S_{*}(\tau)$, the first term on the left-hand side of (8.4) is bounded and the third term tends to $-\infty$. Hence, $\lim _{S \uparrow S_{*}(\tau)} \log (\delta(S)-S) /(1-S)=-\infty$. We thus know

$$
\lim _{S \uparrow S_{*}(\tau)} \delta(S)=S_{*}(\tau) \text { and } \lim _{S \uparrow S_{*}(\tau)} S \eta(S) / d_{\tau}(S)=1
$$

The differentiation of $F\left(S, d_{\tau}(S), v_{\tau}(S)\right)=0$ by $S$ leads to

$$
\begin{aligned}
\log \frac{d_{\tau}+S-v_{\tau}}{1+S}+\log \frac{d_{\tau}-S+v_{\tau}}{1-S}-2 & +\frac{1+S}{d_{\tau}+S-v_{\tau}}\left(d_{\tau}{ }^{\prime}+1-v_{\tau}{ }^{\prime}\right) \\
& -\frac{1-S}{d_{\tau}-S+v_{\tau}}\left(d_{\tau}{ }^{\prime}-1+v_{\tau}{ }^{\prime}\right)=0
\end{aligned}
$$

where $d_{\tau}=d_{\tau}(S), d_{\tau}{ }^{\prime}=d_{\tau}{ }^{\prime}(S), v_{\tau}=v_{\tau}(S)$ and $v_{\tau}{ }^{\prime}=v_{\tau}{ }^{\prime}(S)$. Multiplying (8.5) by $d_{\tau}-S+v_{\tau}$, we obtain

$$
\left(d_{\tau}-S+v_{\tau}\right)\left\{\log \frac{d_{\tau}+S-v_{\tau}}{1+S}+\log \frac{d_{\tau}-S+v_{\tau}}{1-S}-2\right\}
$$

$$
+2 \frac{S-S \eta / d_{\tau}}{1+S \eta / d_{\tau}} d_{\tau}{ }^{\prime}+2 \frac{1-S^{2} \eta / d_{\tau}}{1+S \eta / d_{\tau}}\left(1-v_{\tau}{ }^{\prime}\right)=0
$$

where $\eta=\eta(S)$. Now, by the use of $\lim _{S \uparrow S_{*}(\tau)} S \eta(S) / d_{\tau}(S)=1$ and the fact

$$
0<d_{\tau}-S+v_{\tau}<d_{\tau}+S-v_{\tau} \rightarrow 0 \text { as } S \uparrow S_{*}(\tau)
$$

which follows from (4.8), we succeed to show

$$
\lim _{S \uparrow S_{*}(\tau)} d_{\tau}^{\prime}(S)=1-\lim _{S \uparrow S_{*}(\tau)} v_{\tau}^{\prime}(S)=1-(p \tau)^{-2} .
$$


This together with (3.2) completes the proof of the second property of (4.9).

We next turn to the properties $(4.10) \sim(4.12)$ of $d_{\tau}(S)$ in the limit $S \downarrow 0$. Using

$$
\lim _{S \downarrow 0} \eta(S)=1-p \tau, \quad \lim _{S \downarrow 0} \eta^{\prime}(S)=0 \quad \text { and } \quad \lim _{S \downarrow 0} \eta^{\prime \prime}(S)=-p \tau\left(1-\tau^{2}\right),
$$

we prove the key property $d_{\tau}(S)=O(1)$ around $S=0$. Fix a small positive constant $\delta$ so that $1 / \delta+\log \delta+\log (1-p \tau)-1>0$. It holds that

$$
\begin{aligned}
& F\left(S, \delta \eta(S), v_{\tau}(S)\right)=(1+S) \log \frac{\delta+S}{1+S}-(1-S) \log \frac{\delta-S}{1-S}+2 S \log \eta(S) \\
& =\log \frac{\delta+S}{\delta-S}+S \log (\delta+S)(\delta-S)+2 S \log \eta(S) \\
& -(1+S) \log (1+S)+(1-S) \log (1-S) \\
& =S \cdot G(\delta, S) \text {, } \\
& G(\delta, S)=\frac{1}{S} \log \frac{\delta+S}{\delta-S}+\log \frac{\delta+S}{\delta-S}+2 \log \eta(S)-2+O\left(S^{2}\right) \quad \text { around } S=0 .
\end{aligned}
$$

Since $G(\delta, S)$ tends to $2\{1 / \delta+\log \delta+\log (1-p \tau)-1\}>0$ as $S \downarrow 0$ by (8.8), we know $F\left(S, \delta \eta(S), v_{\tau}(S)\right)=S \cdot G(\delta, S)>0$ for small $S>0$. This together with (8.3) leads to $d_{\tau}(S)>\delta \eta(S)>\delta(1-p \tau) / 2>0$ near $S=0$. We thus obtain $d_{\tau}(S)=O(1)$ around $S=0$. 0 as

Because of $S \eta(S) / d_{\tau}(S)=O(S)$ around $S=0$ we can rewrite $F\left(S, d(S), v_{\tau}(S)\right)=$

$$
\begin{aligned}
0= & (1+S) \log \frac{d_{\tau}\left(1+S \eta / d_{\tau}\right)}{1+S}-(1-S) \log \frac{d_{\tau}\left(1-S \eta / d_{\tau}\right)}{1-S} \\
= & 2 S \log d_{\tau}-(1+S) \log (1+S)+(1-S) \log (1-S) \\
& \quad+(1+S) \log \left(1+S \eta / d_{\tau}\right)-(1-S) \log \left(1-S \eta / d_{\tau}\right) \\
= & 2 S\left(\log d_{\tau}-1+\eta / d_{\tau}\right)+S^{3} \zeta+O\left(S^{4}\right)
\end{aligned}
$$

around $S=0$, where $d_{\tau}=d_{\tau}(S), \eta=\eta(S)$ and $\zeta=\zeta(S)=\left\{1-3\left(\eta / d_{\tau}\right)^{2}+\right.$ $\left.2\left(\eta / d_{\tau}\right)^{3}\right\} / 3$. Multiplying the above equality by $1 / 2 S$, we obtain

$$
0=\log d_{\tau}-1+\eta / d_{\tau}+S^{2} \zeta / 2+O\left(S^{3}\right) \text { around } S=0
$$

which and (8.8) imply the first equality of (4.10). The differentiation of (8.9) yields

$$
0=d_{\tau}{ }^{\prime} / d_{\tau}+\eta^{\prime} / d_{\tau}-\eta d_{\tau}{ }^{\prime} / d_{\tau}^{2}+S \zeta+O\left(S^{2}\right) \text { around } S=0
$$

where $d_{\tau}{ }^{\prime}=d_{\tau}{ }^{\prime}(S)$ and $\eta^{\prime}=\eta^{\prime}(S)$. We thus obtain (4.10) with the aid of (8.8).

Differentiating (8.10) again and taking the limit $S \downarrow 0$, we have

$$
d_{\tau}(0) d_{\tau}^{\prime \prime}(0)+d_{\tau}(0) \eta^{\prime \prime}(0)-\eta(0) d_{\tau}^{\prime \prime}(0)+d_{\tau}(0)^{2} \zeta(0)=0
$$

where $\eta(0)=\lim _{S \downarrow 0} \eta(S), \eta^{\prime \prime}(0)=\lim _{S \downarrow 0} \eta^{\prime \prime}(S)$ and $\zeta(0)=\lim _{S \downarrow 0} \zeta(S)$. We now obtain (4.11) from (8.11) with the aid of (4.10) and (8.8).

Put $q(x)=1-x+x \log x$ and

$$
r(x)=x-x(1-\log x)^{2}(1+2 \log x)-3 q(x)\left(1-q(x)^{2} / p^{2}\right) \quad \text { for } x \in(0,1) .
$$


Then $d_{\tau}{ }^{\prime \prime}(0)$ has the same sign as $r\left(d_{\tau}(0)\right)$. Since $\lim _{x \downarrow 0} r(x)=-3\left(1-1 / p^{2}\right)<0$ and $\lim _{x \uparrow 1} r(x)=0$, it suffices for the proof of (4.12) to show that there exists a unique $x_{c} \in(0,1)$ satisfying $r^{\prime}(x)>0$ if $x<x_{c}$ and $r^{\prime}(x)<0$ if $x>x_{c}$. It holds that $q^{\prime}(x)=$ $\log x<0$ and $r^{\prime}(x)=r_{0}(x) \log x$, where $r_{0}(x)=3\left(1+3 q(x)^{2} / p^{2}\right)-3 \log x-2(\log x)^{2}$. Let $\alpha=e^{\beta}$, where $\beta$ is the smaller solution of $3-3 x-2 x^{2}=0$. Then, if $x<\alpha$ then

$$
\begin{aligned}
x r_{0}{ }^{\prime}(x) & =\left(18 / p^{2}\right) x(1-x+x \log x) \log x-3-4 \log x \\
& >18 x(1-x) \log x-3-4 \log x>-(4-18 \alpha(1-\alpha)) \log x-3>0,
\end{aligned}
$$

and if $x>\alpha$ then $r_{0}(x)>3-3 \log x-2(\log x)^{2}>0$, which implies the unique existence of $x_{c} \in(0, \alpha)$.

(4) Proof of Proposition 5 When $S \in(0,1)$ is fixed, $v_{\tau}(S)$ increases with $\tau$ and its infimum and supremum are 0 and $p$, respectively. Because of the bistable condition $p>1-d$, the infimum $v_{\text {inf }}$ and supremum $v_{\text {sup }}$ of the argument $v$ of $F(S, d, v)$ in (4.6) are given respectively by

$$
v_{\text {inf }}=\max \{0, S-d\} \text { and } v_{\text {sup }}=\min \{1-d, d+S\} .
$$

Since $F(S, d, v)$ decreases with increasing $v$, it suffices for the proof of the unique existence of solution to show

$$
\lim _{v \downarrow v_{\text {inf }}} F(S, d, v)>0 \text { and } \lim _{v \uparrow v_{\text {sup }}} F(S, d, v)<0 .
$$

Assume $v_{\text {inf }}=0$. Then, we have

$$
\lim _{v \downarrow v_{\text {inf }}} F(S, d, v)=(1+S) \log \frac{d+S}{1+S}-(1-S) \log \frac{d-S}{1-S}
$$

which is a monotone decreasing function of $d \in(S, 1)$ tending 0 as $d \uparrow 1$. Hence $\lim _{v \downarrow v_{\text {inf }}} F(S, d, v)>0$. Moreover, if $v_{\text {inf }}=S-d$ then $\lim _{v \downarrow v_{i n f}} F(S, d, v)=\infty$. We thus obtain the first assertion of (8.12). The second assertion is obtained also by noting that

$$
\lim _{v \uparrow v_{\text {sup }}} F(S, d, v)=(1+S) \log \frac{2 d+S-1}{1+S}<0 \text { if } \quad v_{\text {sup }}=1-d
$$

and $\lim _{v \uparrow v_{\text {sup }}} F(S, d, v)=-\infty$ if $v_{\text {sup }}=d+S$.

The property (4.13) follows immediately from the facts

$$
S-d<v_{\tau_{d}(S)}(S)<1-d \text { and } \tau_{d}(S)=\frac{v_{\tau_{d}(S)}(S)}{S}\left\{\frac{1-S^{2}}{p^{2}-v_{\tau_{d}(S)}(S)^{2}}\right\}^{1 / 2} .
$$

Put $v_{\tau_{d}(S)}(S)=S-d+\eta(S)$. Clearly, $0<\eta(S)<1-S$ and $\eta(S)$ tends to 0 as $S \uparrow 1$ by (4.13). Substituting the above $v_{\tau_{d}(S)}(S)$ into (4.6), we have

$$
(1+S) \log \frac{2 d-\eta}{1+S}=(1-S) \log \frac{\eta}{1-S}
$$

where $\eta=\eta(S)$. Since the left-hand side of (8.13) tends to $2 \log d$ as $S \uparrow 1, \eta(S) /(1-S)$ tends to 0 as $S \uparrow 1$. Differentiating (8.13) by $S$ and using (8.13) again, we obtain

$$
\eta^{\prime}(S)=-\frac{2 d-\eta}{S+(1-S) \frac{d}{\eta}}+\frac{2 d-\eta}{S+(1-S) \frac{d}{\eta}} \cdot \frac{1}{1+S} \log \frac{\eta}{1-S},
$$


which tends to 0 as $S \uparrow 1$. We thus obtain (4.14). We omit the proof of (4.15) since it is obtained by applying a similar argument as in the proof of Proposition 4 .

(5) Proof of Proposition 6 We first consider the case where $\tau$ is fixed. Choose $d \in(0,1)$ so that $\tau_{\text {static }}(d)=\tau$. When $S$ is sent to $0, d_{\tau}(S)$ tends to $d, \dot{c}(V(-m))$ to $-2 / p$, both $V^{\prime}(-m)$ and $-V^{\prime}(m)$ to $1-d$ by $(5.3), \kappa$ to $(1+\lambda)^{1 / 2}$ by $(5.7)$ and $2 m$ to $-\log d$ by (5.10). Similarly, when $d$ is fixed and $S$ is sent to $0, \tau_{d}(S), \dot{c}(V(-m)$ ), both $V^{\prime}(-m)$ and $-V^{\prime}(m), \kappa$ and $2 m$ tend to $\tau_{\text {static }}(d),-2 / p, 1-d,(1+\lambda)^{1 / 2}$ and $-\log d$, respectively. We thus know that (5.8) tends to (5.11) as $S \downarrow 0$, and complete the proof. $\square$

(6) Proof of Proposition 7 It follows from (5.3), (5.7), (5.9) and (5.12) that

$$
\begin{gathered}
2 \kappa(0)^{3} \hat{\tau}=p \tau\left(\tau^{2} S^{2}+1-S^{2}\right)^{-3 / 2}=\frac{\partial v_{\tau}}{\partial S}(S), \\
\kappa(0) H_{-}(0)=1-\kappa(0) V^{\prime}(-m)=e^{-2 m \kappa_{+}(0)}=\left(d+S-v_{\tau}(S)\right) /(1+S), \\
\kappa(0) H_{+}(0)=1+\kappa(0) V^{\prime}(m)=e^{2 m \kappa_{-}(0)}=\left(d-S+v_{\tau}(S)\right) /(1-S) .
\end{gathered}
$$

Using $\dot{\kappa}=d \kappa / d \rho=1 /(2 \kappa)$, we obtain

$$
\dot{H}(\rho)=\left(\hat{\tau}-\kappa(\rho)^{-3} / 2\right)\left(H_{-}(\rho)+H_{+}(\rho)\right)+\kappa(\rho)^{-2}(1+2 m \kappa(\rho)) H_{0}(\rho) .
$$

The substitution of $\rho=0$ into the above yields

$$
2 \kappa^{4} \dot{H}(0)=\left(2 \kappa^{3} \hat{\tau}-1\right)\left(\kappa H_{-}+\kappa H_{+}\right)+2(1+2 m \kappa)\left(\kappa H_{-}\right)\left(\kappa H_{+}\right)
$$

with the aid of $H_{0}(0)=H_{-}(0) H_{+}(0)$, where $\kappa=\kappa(0)$ and $H_{ \pm}=H_{ \pm}(0)$. The above equality (8.15) is rewritten as

$$
\frac{2 \kappa^{2}}{H_{-} H_{+}} \dot{H}(0)=\left(\frac{\partial v_{\tau}}{\partial S}(S)-1\right)\left(\frac{1}{\kappa H_{-}}+\frac{1}{\kappa H_{+}}\right)+2-\log \kappa H_{-}-\log \kappa H_{+}
$$

by the use of (8.14). By using (8.14) again, we can rewrite (8.5) as

$$
\begin{aligned}
& \left(\frac{1}{\kappa H_{-}}-\frac{1}{\kappa H_{+}}\right) d_{\tau}{ }^{\prime}(S) \\
& \quad=\left(\frac{\partial v_{\tau}}{\partial S}(S)-1\right)\left(\frac{1}{\kappa H_{-}}+\frac{1}{\kappa H_{+}}\right)+2-\log \kappa H_{-}-\log \kappa H_{+} .
\end{aligned}
$$

Fix $d \in(0,1)$ and put $\tau=\tau_{d}(S)$ in (4.6). Differentiating the resulting equality by $S$, we get

$$
-\frac{\partial v_{\tau}}{\partial \tau}(S)\left(\frac{1}{\kappa H_{-}}+\frac{1}{\kappa H_{+}}\right) \tau_{d}^{\prime}(S)=\text { the same as the right-hand side of (8.17). }
$$

All the right-hand sides of $(8.16),(8.17)$ and (8.18) are the same. The coefficient of $\dot{H}(0)$ is positive while those of $d_{\tau}{ }^{\prime}(S)$ and $\tau_{d}{ }^{\prime}(S)$ are negative. We thus complete the proof.

(7) Proof of Proposition 8 (1) Clearly $\lim _{\rho \uparrow \infty} H(\rho)=\infty$. Since $\dot{H}(0)<0$ by Proposition 7 , the equation $H(\rho)=0$ has a positive solution and (5.8) has a positive eigenvalue.

(2) From the equality $\dot{\kappa}=1 /(2 \kappa)>0$, we have $d H_{0} / d \rho(\rho)<0$ for any $\rho>-1$. Moreover, $d H_{-} / d \rho=d H_{+} / d \rho=\hat{\tau}-\kappa^{-2} \dot{\kappa}=\hat{\tau}-\kappa^{-3} / 2$ and $\hat{\tau}-\kappa^{-3} / 2$ increases with 
$\rho$. Hence, if $\hat{\tau}-\kappa(0)^{-3} / 2 \geq 0$ then (5.4) has no non-negative real eigenvalue except the 0-eigenvalue corresponding to the translation invariance. By (5.3) and (5.7), the condition $\hat{\tau}-\kappa(0)^{-3} / 2 \geq 0$ is equivalent to

$$
p \tau \geq\left(\tau^{2} S^{2}+1-S^{2}\right)^{3 / 2} .
$$

Since the right-hand side of (8.19) tends to $(p \tau)^{3}$ when $\tau<1 / p$ is fixed and $S \uparrow S_{*}(\tau)$, (8.19) is satisfied. When $d$ is fixed, $\tau=\tau_{d}(S)=O\left((1-S)^{1 / 2}\right)$ around $S=1$ by (4.13) and (4.14). Hence, the left-hand side of $(8.19)$ is of $O\left((1-S)^{1 / 2}\right)$ while the right-hand side is of $O\left((1-S)^{3 / 2}\right)$ around $S=1$, and (8.19) is satisfied.

(8) How to calculate the Hopf bifurcation point of standing pulse Put $\kappa=\alpha+i \beta(\alpha \in \mathbf{R}, \beta \in \mathbf{R}$ and $i=\sqrt{-1})$ in (3.5). Then $\lambda=\alpha^{2}-\beta^{2}-1+2 i \alpha \beta$. The Hopf bifurcation point $\tau_{H}(d)$ is determined so that (3.5) has a pair of pure imaginary solutions, and in this case (3.5) is split into the following real and imaginary parts:

$$
\begin{aligned}
& 0=1-d-\left\{\alpha+e^{-2 m \alpha}(\alpha \cos 2 m \beta-\beta \sin 2 m \beta)\right\} /\left(\alpha^{2}+\beta^{2}\right), \\
& p \alpha \beta \tau_{H}(d)=\left\{\beta+e^{-2 m \alpha}(\beta \cos 2 m \beta+\alpha \sin 2 m \beta)\right\} /\left(\alpha^{2}+\beta^{2}\right),
\end{aligned}
$$

where $\beta=\beta(\alpha)=\left(\alpha^{2}-1\right)^{1 / 2}$. Lemma 3.11 in [10] for a class of more general nonlinearities pointed out that the right-hand side of (8.20) increases with $\alpha$. Moreover, the right-hand side of (8.20) tends to $-2 d<0$ (resp. $1-d>0$ ) as $\alpha \downarrow 1$ (resp. $\alpha \uparrow \infty)$. Hence, we can calculate the solution $\alpha$ of (8.20) by the bisection method and get $\tau_{H}(d)$ from (8.21).

(9) How to calculate the branch of travelling pulses As stated in the proof of Proposition $4, d_{\tau}(S)$ is a unique zero-point of the monotone decreasing function $F\left(S, d, v_{\tau}(S)\right)$ of $d$. Hence we can calculate $d_{\tau}(S)$ by the bisection method. The turning point $Q_{\text {turn }}(\tau)$ is, if exists, calculated by applying the bisection method to $d_{\tau}{ }^{\prime}$ expressed as (8.5). Since $\tau_{d}(S)$ is also a unique zero-point of the monotone decreasing function $F\left(S, d, v_{\tau}(S)\right)$ of $\tau$ as described in the proof of Proposition $5, \tau_{d}(S)$ can be calculated by the bisection method.

(10) How to calculate eigenvalues along the branch of travelling pulses We denote by $H(\lambda ; S, \tau)$ the function defined by the left-hand side of (5.8), and the 0 -eigenvalue corresponding to phase shifts by $\lambda_{0}$. We explain the method in the case where $\tau$ is fixed.

Real eigenvalues $\lambda$ are real roots of (5.8). We began with the observation of graphs of $H(\lambda ; S, \tau)$, which are classified into five typical patterns as shown in Figure 8.1. There seems to exist a constant $S_{\text {complex }}$ such that (5.8) has four simple real roots $\lambda_{1}<\lambda_{2}<\lambda_{3}<\lambda_{0}$ for $S>S_{\text {complex }}$ (the case (1) of Figure 8.1), two simple real roots $\lambda_{1}<\lambda_{0}$ and one double root $\lambda_{2}=\lambda_{3}$ for $S=S_{\text {complex }}$ (the case (2)), two real roots $\lambda_{1}, \lambda_{0}$ and one pair of complex conjugate roots for $S<S_{\text {complex }}$ (the cases $(3) \sim(5))$. When $S$ is near $S_{*}(\tau)$, the graph is like (1) and (5.8) has four real roots $\lambda_{1}<\lambda_{2}<\lambda_{3}<\lambda_{0}$. As $S \uparrow S_{*}(\tau)$ both $\lambda_{1}$ and $\lambda_{2}$ tend to the same negative number and $\lambda_{3}$ to 0 from the below. When the branch of travelling pulses has a turning point $Q_{t u r n}(\tau)$, the graph changes its shape like (1), (2), (3), (4) and (5) in the order as $S$ decreases, and $\lambda_{1}$ tends to 0 from the above as $S \downarrow 0$. When the branch has no turning point, the shape changes like (1), (2) and (3) in the order as $S$ decreases, and $\lambda_{1}$ tends to 0 from the below as $S \downarrow 0$.

If we find some $S_{0}$ such that $H\left(\lambda ; S_{0}, \tau\right)=0$ has four simple roots and initial values $\rho_{0}, \rho_{1}, \rho_{2}$ and $\rho_{3}$ satisfying $-1<\rho_{0}<\lambda_{1}<\rho_{1}<\lambda_{2}<\rho_{2}<\lambda_{3}<\rho_{3}<\lambda_{0}$ for 


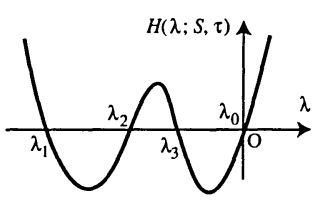

(1)

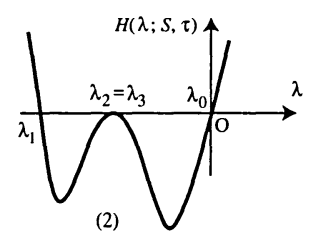

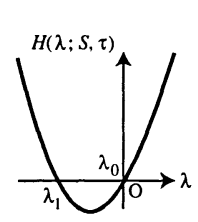

(3)

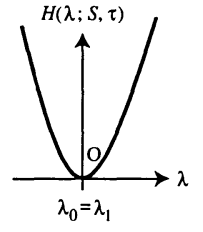

(4)

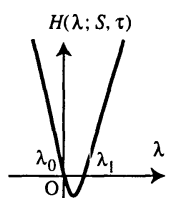

(5)

FIG. 8.1. Typical graphs of $H(\lambda ; S, \tau)$ for several $S$

its unknown roots $\lambda_{1}, \lambda_{2}$ and $\lambda_{3}$, then the roots are estimated by the bisection method. Moreover, once we get nice approximated roots $\hat{\lambda}_{1}, \hat{\lambda}_{2}$ and $\hat{\lambda}_{3}$ of $H\left(\lambda ; S_{0}, \tau\right)=0$, then $\left(\hat{\lambda}_{1}-1\right) / 2,\left(\hat{\lambda}_{1}+\hat{\lambda}_{2}\right) / 2,\left(\hat{\lambda}_{2}+\hat{\lambda}_{3}\right) / 2$ and $\hat{\lambda}_{3} / 2$ become excellent initial values in order to find the roots of $H(\lambda ; S, \tau)=0$ for $S$ belonging to a neighborhood of $S_{0}$.

We next proceed to complex eigenvalues. We have effectively calculated them based on the continuity of (5.8) and (5.11) at the static bifurcation point of standing pulses. Putting $\kappa=\kappa(\lambda)=\alpha+i \beta(\alpha \in \mathbf{R}, \beta \in \mathbf{R}$ and $i=\sqrt{-1})$, we split each of (5.8) and (5.11) into the real and imaginary parts. We thus obtain a system of transcendental equations with respect to $\alpha$ and $\beta$, say, $H_{t p}(\alpha, \beta ; S, \tau)=0$ from (5.8) and $H_{s p}(\alpha, \beta ; d, \tau)=0$ from (5.11). Giving an approximated solution $d_{0}$ of $\tau_{H}(d)=\tau$, we calculate the solution $\left(\tau_{H}\left(d_{0}\right), \alpha_{t m p}, \beta_{t m p}\right)$ of (8.20) and (8.21) with $d=d_{0}$, where $\alpha_{t m p}>1$ and $\beta_{t m p}=\left(\alpha_{t m p}^{2}-1\right)^{1 / 2}$. After these steps we get the solution $\left(\alpha_{0}, \beta_{0}\right)$ of $H_{s p}\left(\alpha, \beta ; d_{0}, \tau\right)=0$ by using Newton's method and adopting $\left(\alpha_{t m p}, \beta_{t m p}\right)$ as an initial value. Let $d_{j}=d_{0}+j\left(d_{\tau}(0)-d_{0}\right) / M(j=1, \cdots, M)$ and $0=S_{0}<S_{1}<\cdots<$ $S_{N}<S_{*}(\tau)$, where $M$ and $N$ are positive integers. Newton's method with the initial value $\left(\alpha_{j-1}, \beta_{j-1}\right)$ gives the solution $\left(\alpha_{j}, \beta_{j}\right)$ of $H_{s p}\left(\alpha, \beta ; d_{j}, \tau\right)=0$ iteratively for $j=1, \cdots, M$. Then, $\left(\alpha_{M}, \beta_{M}\right)$ satisfies also $H_{t p}(\alpha, \beta ; 0, \tau)=0$, which is equivalent to $H_{s p}\left(\alpha, \beta ; d_{\tau}(0), \tau\right)=0$ since (5.8) and (5.11) are continuous at the static bifurcation point of standing pulses. The successive application of Newton's method with initial value $\left(\alpha_{M+k-1}, \beta_{M+k-1}\right)$ yields the solution $\left(\alpha_{M+k}, \beta_{M+k}\right)$ of $H_{t p}\left(\alpha, \beta ; S_{k}, \tau\right)=0$ $(k=1, \cdots, N)$.

Let $(\alpha(S), \beta(S))$ be the solution of $H_{t p}(\alpha, \beta ; S, \tau)=0$. Then this pair satisfies $\alpha(S)^{2}-\beta(S)^{2}-1=0$ at the Hopf bifurcation point $Q_{H o p f}(\tau)$. Hence, $S$-component of $Q_{\text {Hopf }}(\tau)$ is calculated by applying the bisection method to $\alpha(S)^{2}-\beta(S)^{2}-1=0$.

\section{REFERENCES}

[1] T. AokI, The Existence Region of Stable Travelling Breathers, Master's thesis, Ryukoku University, 1996 (in Japanese).

[2] J. D. DockERY, Existence of standing pulse solutions for an excitable activator-inhibitory system, J. Dynamics and Differential Equations, 4 (1992), pp. 231--257.

[3] J. D. Dockery AND J. P. KeEner, Diffusive effects on dispersion in excitable media, SIAM J. Appl. Math., 49 (1989), pp. 539-566. 
[4] R. J. Field AND M. Burger, Oscillation and Travelling Waves in Chemical Reaction, Wiley, New York, 1985.

[5] G. B. Ermentrout, S. P. Hastings, and W. C. Troy, Large amplitude stationary waves in an excitable lateral-inhibition medium, SIMA J. Appl. Math., 44 (1984), pp. 1133-1149.

[6] D. HenRY, Geometric Theory of Semilinear Parabolic Equations, Lecture Notes in Math. 80, Springer-Verlag, New York, 1981.

[7] H. IKEDA, Singular perturbation approach to stability properties of traveling wave solutions of reaction-diffusion systems, Hiroshima Math. J., 19 (1989), pp. 587-630.

[8] H. IKEDA, Singular pulse wave bifurcations from front and back waves in bistable reactiondiffusion systems, Methods and Applications of Analysis, 3 (1996), pp. 285-317.

[9] H. IKEDA, Existence and stability of pulse waves bifurcated from front and back waves in bistable reaction-diffusion systems, Japan J. Indust. Appl. Math., 15 (1998), pp. 163-231.

[10] H. IKEDA AND T. IKEDA, Bifurcation phenomena from standing pulse solutions in some bistable reaction-diffusion systems, J. Dynamics and Differential Equations, 12 (2000), pp. 117-167.

[11] H. IKEDA, M. MimurA, AND Y. NishiURA, Global bifurcation phenomena of traveling wave solutions for some bistable reaction-diffusion systems, Nonlinear Anal. TMA, 13 (1989), pp. 507-526.

[12] T. IKEDA, Numerical simulation for nonlinear oscillation of internal layers, in Advances in Numerical Mathematics, T. Ushijima, Z. Shi, and T. Kako, ed., Lecture Notes in Num. Appl. Anal. 14, Kinokuniya, Tokyo, 1995, pp. 69-77.

[13] T. Ikeda and Y. Nishiura, Pattern selection for two breathers, SIAM J. Appl. Math., 54 (1994), pp. 195-230.

[14] H. KoKubu, Y. Nishiura, AND H. OKA, Heteroclinic bifurcations in bistable reaction-diffusion systems, J. Differential Equations, 86 (1990), pp. 260-341.

[15] Y. Kuramoto, Chemical Oscillations, Waves and Turbulence, Springer Series on Synergetics 19, Springer-Verlag, 1984.

[16] H. P. MCKean, Nagumo equation, Adv. Math., 4 (1970), pp. 209-223.

[17] M. Mimura, M. NAGayama, H. IKedA, AND T. IKedA, Dynamics of travelling breathers arising in reaction-diffusion systems - ODE modelling approach -, accepted by Hiroshima Math. J..

[18] Y. Nishiura, Singular limit approach to stability and bifurcation for bistable reaction-diffusion systems, Rocky Mountain J. Math., 21 (1991), pp. 727-767.

[19] Y. NishiURA AND M. MimURA, Layer oscillations in reaction-diffusion systems, SIAM J. Appl. Math., 49 (1989), pp. 485-514.

[20] Y. Nishiura, M. Mimura, H. IKeda, AND H. Fujil, Singular limit analysis of stability of traveling wave solutions in bistable reaction-diffusion systems, SIAM J. Math. Anal., 21 (1990), pp. 85-122.

[21] J. J. TYSON AND P. C. FIFE, Target patterns in a realistic model of the Belousov-Zhabotinskii reaction, J. Chem. Phys., 73 (1980), pp. 2224-2237.

[22] A. T. Winfree, Rotating chemical reactions, Sci. Amer., 230 (1974), pp. 82-95. 
